\title{
Therapeutic efficacy and safety of radiofrequency ablation for the treatment of trigeminal neuralgia: a systematic review and meta-analysis
}

This article was published in the following Dove Medical Press journal: Journal of Pain Research

\author{
Huiqun $\mathrm{Wu}^{1, *}$ \\ Ju Zhou ${ }^{1, *}$ \\ Jialu Chen' \\ Yuwei Gu' \\ Lili Shi' \\ Haosheng $\mathrm{Ni}^{2}$ \\ 'Department of Medical Informatics, \\ Medical School of Nantong \\ University, Nantong 22600I, People's \\ Republic of China; ${ }^{2}$ Department \\ of Otorhinolaryngology, Affiliated \\ Hospital of Nantong University, \\ Nantong 22600I, People's Republic \\ of China
}

*These authors contributed equally to this work

\begin{abstract}
Objective: The objective of this study was to summarize the effectiveness and safety of trigeminal neuralgia (TN) treatment via different radiofrequency approaches such as continuous radiofrequency (CRF), pulsed radiofrequency (PRF), and combined CRF and pulsed radiofrequency (CCPRF) treatments, thus providing high-quality clinical evidence for TN treatment.

Methods: A series of databases were searched for relevant articles published between January 1998 and April 2018. The modified Jadad scale was referred to evaluate the methodological quality of the included studies. Data were extracted independently, and the outcome and safety of different routes, temperatures, and guidance used in CRF, PRF, and CCPRF were compared. Meta-analysis and publication bias were calculated using Review Manager software.

Results: In total, 34 studies involving 3,558 participants were included. With regard to TN treatment, PRF had no difference in cured rate in comparison with CRF, while CRF was more effective than CCPRF $(P<0.05)$. The comparison of complication rates showed that PRF and CCPRF were safer. For puncture guidance via $\mathrm{CRF}$, three-dimensional-printed template was more accurate in success rate at first puncture than computed tomography guidance $(P<0.05)$. For puncture route, foramen rotundum (FR) or pterygopalatine fossa (PPF) route had no significance in efficiency rate via CRF in comparison with foramen oval (FO) route, but PPF and FR routes were safer. For $\mathrm{CRF}$ treatment, low temperature $\left(68^{\circ} \mathrm{C}-70^{\circ} \mathrm{C}\right)$ compared with high temperature $\left(71^{\circ} \mathrm{C}-75^{\circ} \mathrm{C}\right)$ had no effect. Moreover, higher temperature $\left(66^{\circ} \mathrm{C}-80^{\circ} \mathrm{C}\right)$ had a greater effect compared with lower temperature $\left(55^{\circ} \mathrm{C}-65^{\circ} \mathrm{C}\right)$ on $\mathrm{TN}$ treatment $(P<0.05)$, while the safety of which was decreasing. Conclusion: CCPRF could achieve a greater effect and safety on TN treatment. FR and FO routes in TN puncture treatment via CRF are safer. Medium temperature range is better for CRF therapy, and higher temperature is recommended in PRF, especially for the elders. Further international multicenter trials are needed to confirm the evidence.
\end{abstract}

Keywords: trigeminal neuralgia, radiofrequency ablation, radiofrequency thermocoagulation, meta-analysis

\section{Introduction}

Neuropathic pain is a kind of painful experience, which is often associated with a specific condition. There are several types of neuropathic pain, such as postherpetic neuralgia, trigeminal neuralgia (TN), painful diabetic peripheral neuropathy, and glossopharyngeal neuralgia. Among them, $\mathrm{TN}$ is a clinically common painful disease and was estimated to account for 12.6-28.9/100,000 in the general population per year. ${ }^{1}$ It is a recurrent intense paroxysmal pain in the facial trigeminal area which is like knife-cutting, acupuncture, electric shock, or cauterization. Its frequency and degree of pain increase with the duration of the disease.
Correspondence: Haosheng $\mathrm{Ni}$ Department of Otorhinolaryngology, Affiliated Hospital of Nantong University, 20 Xisi Street, Nantong 22600I, People's Republic of China

$\mathrm{Tel}+865138505$ I89|

Fax+8651385051820

Email entnhs@ntu.edu.cn 
TN can be effectively treated by minimally invasive techniques such as microvascular decompression, ${ }^{2}$ balloon compression, ${ }^{3}$ continuous radiofrequency (CRF) thermocoagulation, and radiosurgery. ${ }^{4}$ In these approaches, CRF is widely used for the clinical treatment of TN with a pain relief rate of 90-100\%. ${ }^{5} \mathrm{CRF}$ could produce heat by vibration and friction, which further leads to thermocoagulation, denaturation, and necrosis of the target tissue. However, patients accepting CRF may develop various complications, such as facial numbness, mouth penetration, eyelash hypoesthesia, forehead numbness, corneal hypoesthesia, and dysacousis. ${ }^{6,7}$ These complications are presumably due to neuronal injury mainly produced by surgical puncture and heat from radiofrequency temperature during the thermocoagulation. ${ }^{8}$ Moreover, the higher the temperature is, the more those complications are. Another method, pulsed radiofrequency (PRF), an ideal technique for the treatment of chronic pain, has been proved as a minimally invasive, safe, and effective interventional treatment choice for TN patients. ${ }^{9-12} \mathrm{PRF}$ uses a lower temperature application of energy generated by the radiofrequency generator at the tip of the needle and transmits the energy to the nerve in a pulse manner. However, its efficacy for pain relief in TN remains controversial. Besides, different temperatures used in those radiofrequency therapy might lead to different outcomes. ${ }^{13-15}$ Meanwhile, the combined CRF and pulsed radiofrequency (CCPRF) has been proposed and verified in clinical trials with pain relief efficiency compared with PRF or CRF treatment alone in patients with chronic pain. ${ }^{16}$

In order to resolve the abovementioned controversies, the aim of this study was to summarize the effectiveness and safety of the treatment of TN via different radiofrequency approaches such as CRF, PRF, and CCPRF treatments. Besides, we compared the outcome and safety of different routes, temperatures, and different kinds of guidance used in above approaches, thus providing high-quality clinical evidence for TN treatment.

\section{Methods}

\section{Search strategy}

We searched the China National Knowledge Infrastructure (CNKI), Chinese VIP Information (VIP), Wanfang, Web of Science, and PubMed databases. Searches that are limited to human studies published between January 1998 and January 2018 were carried out in parallel by YWG and JZ. We used the following search terms: "idiopathic trigeminal neuralgia," "TN," "semilunar ganglion," "gasserian ganglion," “oval foramen," "pulsed," “continuous," "percutaneous," "conventional," "radiofrequency thermocoagulation," "radiofrequency ablation," “CRF,” “PRF,” "RFT,” "CCPRF," "radiofrequency temperature," "treatment," "therapy," "pain relief," "complication*," "recurrence rate," and "satisfact*." The terms were combined with logical connector AND for each component such as patient's condition, intervention, control, and outcome, while OR was used for all candidate terms inside each component. In this way, a subset of citations that address the objective of our research study were generated. The reference lists of relevant articles were hand-searched to get potential eligible studies beyond the electronic searches.

\section{Inclusion criteria}

All clinical studies involving radiofrequency interventions used for the treatment of classical TN were included. The diagnostic criteria for $\mathrm{TN}$ were based on the principle of International Classification of Headache Disorders (International Headache Society [IHS])-II (2004) $)^{17}$ and IHS-III (2013)..$^{18}$ The treatments described were CRF, PRF, and PRF + CRF/CCPRF. Outcome indices were as follows: 1) efficiency of treatment: cured rate, determined as the proportions of patients with $>50 \%$ pain relief, and effective rate, determined as the proportions of patients with $>25 \%$ pain relief; 2) the severity of pain, defined using visual analog scale (VAS) score (0: no pain; $1-3$ : mild pain; 4-6: moderate pain; and 7-10: severe pain) or numerical rating scale (NRS; 0: no pain; 1-3: mild pain; 4-6: moderate pain; 7-9: severe pain, 10: the most painful); 3) satisfactory, determined as the assessment of life quality from 0 (lowest) to 22 (highest) using Life Satisfaction Index B; 4) success rate at the first puncture, determined as first-quantified puncture of effective nerve puncture; and 5) safety: complication rate, determined as the incidence of complications including facial numbness, masticatory muscle weakness, neurological disorder, hematoma, nausea, and vomiting.

\section{Exclusion criteria}

The exclusion criteria were as follows: 1) The subjects of the study were patients accompanied with other diseases affecting TN; 2) studies without the control group; 3) studies about secondary $\mathrm{TN}$ caused by tumors, intracranial lesions, multiple sclerosis, herpes zoster, or other severe organ diseases; 4) insufficient data in outcome index; and 5) the literature with a reporting language other than English and Chinese.

\section{Selection of studies and data extraction}

Three authors (JZ, JLC, and YWG) completed study selection, data extraction, and cross-checking independently 
according to the inclusion and exclusion criteria. We read the titles and abstracts (when available) of all the articles to identify whether it was related to the theme through the searches. Then, we obtained the full articles of these studies and independently judged whether they met the inclusion criteria or not. We resolved any disagreement and difficulties by discussion or consulting another reviewer. The extracted data included demographic data of patients (sample size, sex, age, preoperational pain duration, pain side, and preoperational drug dosage), treatment protocols (methods, temperature, lesion time, stimulating voltage, stereotaxis, and surgery duration), and the efficacy of the treatment including pain score (VAS and NRS), the quality of life (QoL) as well as complications.

\section{Evaluation of methodological quality for the included studies}

The modified Jadad scale ${ }^{19}$ was referred to evaluate the methodological quality of the included studies according to the following four items: 1) the generation of random sequences: i) adequate: random numbers or similar methods generated by computers or random number table (two points); ii) unclear: random test without random distribution method (one point); and iii) inadequate: alternate distribution method, such as odd and even numbers (zero point); 2) randomization: i) adequate: center or pharmacy control allocation scheme, or containers with consistent sequence numbers, onsite computer control, sealed opaque envelopes, or other methods so that clinicians and participants cannot predict the allocation sequence (two points); ii) unclear: only using a random number table or other random allocation scheme (one point); iii) inadequate: alternate distribution, case number, and any other measures cannot prevent predictability packets; and iv) unused (zero point); 3 ) blindness: i) adequate: using completely consistent placebo tablets or similar methods (two points); ii) unclear: just having the statement of blindness, but without description (one point); and iii) inadequate: not by double-blindness or way of blindness is not appropriate, such as the comparison of tablets and injections (zero point); and 4) follow-up: describing the number and reasons for withdrawal (one point), without the number or reasons for withdrawing (zero point). In a word, 1-3 points were considered as low quality, and 4-7 points were considered to be of high quality.

\section{Statistical analysis}

In this study, meta-analysis and publication bias were calculated using Review Manager (RevMan5.3; The Nordic Cochrane Center, The Cochrane Collaboration, Copenhagen,
Denmark). ORs were used for evaluation, and 95\% CIs were calculated for each estimate. Heterogeneity was considered low, moderate, or high for $\mathrm{I}^{2}$ values $<25 \%, 25-50 \%$, and $>50 \%$, respectively. The analyses were performed using a random-effects model on those studies with high heterogeneity $\left(\mathrm{I}^{2}>50 \%\right)$ and with fixed-effects model on those studies with less heterogeneity $\left(\mathrm{I}^{2} \leq 50 \%\right) . P \leq 0.05$ was considered statistically significant.

\section{Results}

The relevant article search yielded 2,142 references from PubMed, Web of Science, CNKI, VIP, and Wanfang databases, of which 34 articles $^{14,15,20-51}$ were qualified for this study finally according to the flowchart. Figure 1 presents the study selection process.

\section{Summary characteristics of the included studies}

All the included studies met inclusion and exclusion criteria. A total of 34 studies involving 3,558 subjects from different regions including Turkey, Egypt, Korea, and People's Republic of China were finally included in this meta-analysis. The age of the included population mainly ranged from 55 to 75 years, and $\mathrm{TN}$ is commonly seen in the middle-aged and elderly people. Each study recruited both men and women in the case and control groups. TN occurred at the left and right sides, and the most suffered areas were on the right side. Meanwhile, most studies suggested that the pain at the two sides were often involved two (V2 + V3) branches, while the pain at one side most commonly involved only one (V2) branch. For the treatment, all studies reported the way of radiofrequency ablation (eg, CRF, PRF, and CCPRF), temperature, surgery time, and guidance (eg, X-ray, computed tomography [CT], type-B ultrasonography, and MRI). In these studies, 19 articles investigated the association between different kinds of guidance and the efficiency of CRF, and six articles reported the association between different routes of thermocoagulation and CRF. Six articles compared the efficiency of CRF by different temperatures, while three articles analyzed the effect of different temperatures in PRF treatment. Preoperational pain duration was partially inconsistent among the involved studies. The outcome index for efficiency and safety involved in different articles were pain score (VAS and NRS), QoL, and complications. The most common complications were facial hematoma, facial numbness, nausea and vomiting, headache, masticatory muscle weakness, hearing loss, facial swelling and congestion, corneal paralysis, and so on. Table 1 lists the details of the included studies. 


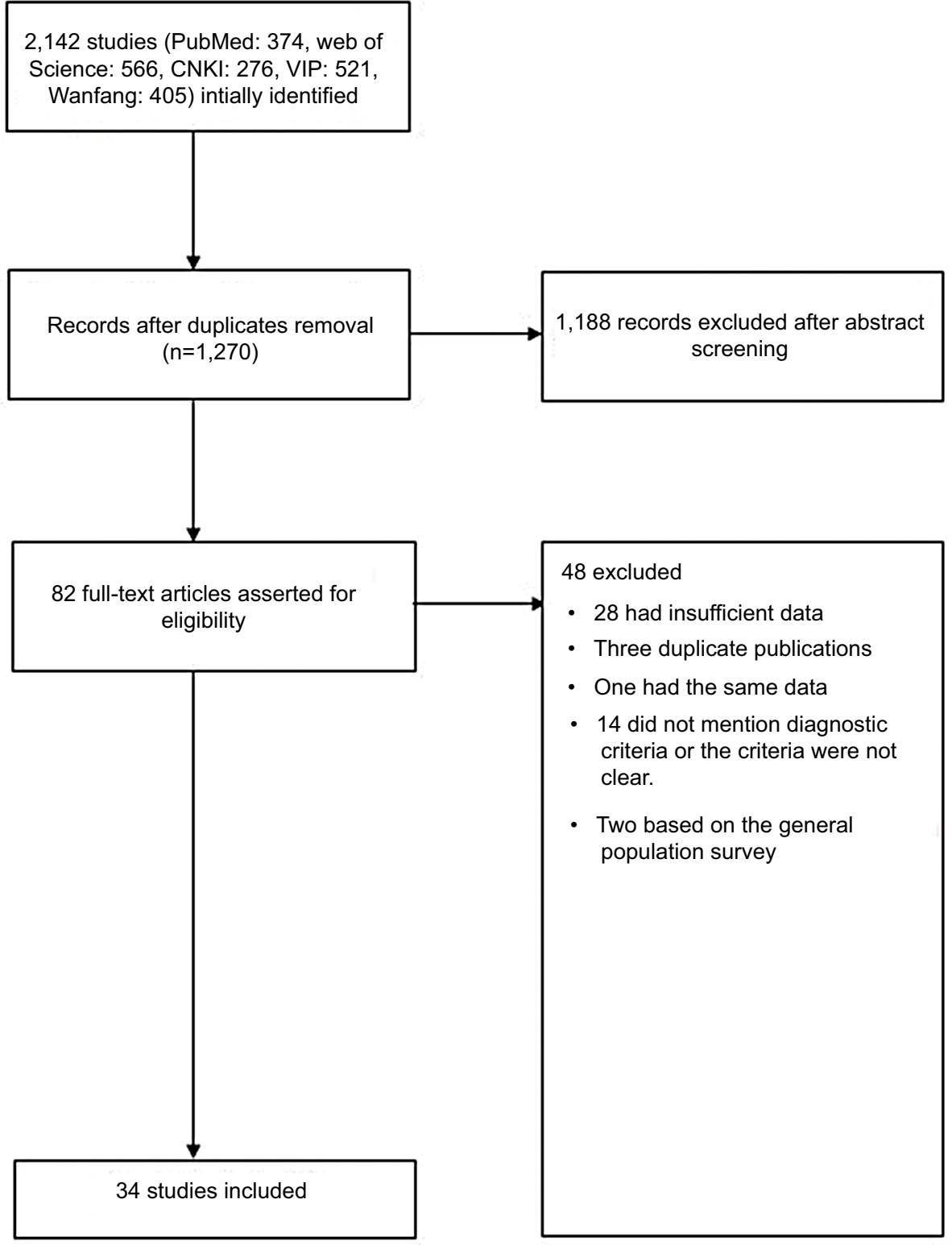

Figure I Flowchart of the study selection.

Abbreviations: CNKI, China National Knowledge Infrastructure; VIP, Chinese VIP Information.

\section{Quality of the included studies}

Most included studies cannot be completely double-blind; as a result, their Jadad scale scores were less than four points. Table 2 shows the detailed scores of each study. In total, $79.4 \%$ (27 of 34) of the included studies were of low quality; the scale of two studies was zero point, nine studies one point, eight studies two points, and eight studies three points. Only seven clinically randomized trials were of high quality.

\section{Comparison of efficiency and safety between PRF and CRF}

Figure 2 shows the comparison of cured rate and complication of TN after thermocoagulation treatments (PRF vs CRF).
Six studies ${ }^{22,24,27,28,32,33}$ in the PRF group compared with the CRF group patients in cured rate showed great heterogeneity $\left(\chi^{2}=20.66, P=0.0009<0.05, \mathrm{I}^{2}=76 \%\right)$; the total effect size OR in this study was 0.60 (95\% CI: $0.19,1.92)$, and the $\mathrm{Z}$ value was $0.86(P=0.39>0.05)$, indicating that there was no statistically significant difference in the cured rate between PRF and CRF in TN treatment. Seven studies ${ }^{22-24,27,28,32,33}$ in the PRF group compared with the CRF group patients in complications of mortality showed great heterogeneity of the studies $\left(\chi^{2}=28.13, P<0.0001, \mathrm{I}^{2}=79 \%\right)$; the total effect size OR in this study was 0.04 (95\% CI: $0.01,0.23$ ), and the $\mathrm{Z}$ value was $3.70(P=0.0002<0.05)$, indicating that $\mathrm{PRF}$ was safer than CRF in TN treatment. 


\section{Comparison of efficiency and safety between CRF and CCPRF}

Figure 3 shows the comparison of cured rate and complication of TN after thermocoagulation treatments (CRF vs CCPRF). Three studies ${ }^{20,25,27}$ in the CRF group compared with the CCPRF group patients in cured rate showed great heterogeneity of the studies $\left(\chi^{2}=6.92, P=0.03<0.05\right.$, $\left.\mathrm{I}^{2}=71 \%\right)$; the total effect size OR in this study was 0.20 (95\% CI: $0.08,0.50$ ), and the $\mathrm{Z}$ value was 3.44 $(P=0.0006<0.05)$. Five studies ${ }^{20,25,27,29,31}$ in the CRF group compared with the CCPRF group patients in complication showed less heterogeneity of the studies $\left(\chi^{2}=6.03\right.$, $\left.P=0.20>0.05, \mathrm{I}^{2}=34 \%\right)$; the total effect size OR in this study was 2.53 (95\% CI: $1.51,4.26)$, and the $Z$ value was $3.51(P=0.0005<0.05)$. All the results suggested that the CCPRF group had a greater effect and was safer compared with the CRF group.

\section{Comparison of the cured rate between three-dimensional (3D) CT guidance vs manual puncture in CRF treatment}

Figure 4 shows the comparison of the cured rate between different kinds of guidance for RF (between 3D CT vs manual puncture) in TN treatment. Two studies ${ }^{40,41}$ in the 3D CT group compared with the manual puncture group patients showed great heterogeneity of the studies $\left(\chi^{2}=2.58\right.$, $\left.P=0.11<0.05, \mathrm{I}^{2}=61 \%\right)$; the total effect size OR in this study was 0.85 (95\% CI: $0.49,1.48)$, and the $Z$ value was 0.57 ( $P=0.57>0.05$ ), suggesting that there was no significance of the cure rate between $3 \mathrm{D} \mathrm{CT}$ guidance vs manual puncture in TN treatment.

\section{Comparison of the success rate at first puncture between 3D-printed guide plate vs $C T$ guidance in CRF treatment}

Figure 5 shows the comparison of the success rate at first puncture between different kinds of guidance for RF (3D-printed guide plate vs CT guide) in TN treatment. Two studies $^{34,35}$ in the 3D-printed guide group compared with the CT guide group patients showed no heterogeneity of the studies $\left(\chi^{2}=0.63, P=0.43>0.05, \mathrm{I}^{2}=0 \%\right)$; the total effect size OR in this study was 55.47 (95\% CI: 12.47, 246.69), and the $Z$ value was $5.27(P=0.00001<0.05)$, indicating that $3 \mathrm{D}$-printed guide has a great effect on treatment in success rate at first puncture.

\section{Comparison of the success rate at first puncture between combined guidance and simple image guidance in CRF treatment}

Figure 6 shows the comparison of the success rate at first puncture between different kinds of guidance for RF (combined with other means vs simple image-guided like CT or C-arm) in TN treatment. Three studies $\mathrm{s}^{36,37,39}$ with combined stimulation potential guide group compared with the simple image-guided group showed no heterogeneity of the studies $\left(\chi^{2}=0.37, P=0.83>0.05\right.$, $\left.\mathrm{I}^{2}=0 \%\right)$; the total effect size $\mathrm{OR}$ in this study was $7.38(95 \% \mathrm{CI}$ : $2.41,22.54)$, and the $Z$ value was $3.51(P=0.0005<0.05)$. Two studies $^{38,42}$ in the CT combined semiconductor laser locator compared with the simple CT guide group patients showed no heterogeneity of the studies $\left(\chi^{2}=0.02, P=0.89>0.05, \mathrm{I}^{2}=0 \%\right.$ ); the total effect size OR in this study was 3.18 (95\% CI: 1.58 , $6.49)$, and the $\mathrm{Z}$ value was $3.19(P=0.001<0.05)$. The results indicated that the guidance combined with other means had a greater success rate than that of the simple image guidance on the treatment of TN patients at first puncture.

\section{Comparison of efficiency and safety between the pterygopalatine fossa (PPF) vs foramen oval (FO) route in TN treatment via CRF}

Figure 7 shows the comparison of the effective rate and complication of different routes (the PPF group vs the FO group) in treating TN. Three studies ${ }^{43,46,47}$ in the PPF group compared with the FO group showed no heterogeneity of the studies $\left(\chi^{2}=0.05, P=0.98>0.05, \mathrm{I}^{2}=0 \%\right)$; the total effect size $\mathrm{OR}$ in this study was 0.87 (95\% CI: $0.30,2.55)$, and the $Z$ value was $0.25(P=0.81>0.05)$, suggesting that there was no significant difference in the therapeutic effect between the PPF route and the FO route in TN treatment via CRF. Three studies ${ }^{43,46,47}$ in the PPF group compared with the FO group showed no heterogeneity of the studies $\left(\chi^{2}=0.24, P=0.89>0.05, \mathrm{I}^{2}=0 \%\right)$; the total effect size OR in this study was 0.06 (95\% CI: 0.01 , $0.27)$, and the $Z$ value was $3.65(P=0.0003<0.05)$, suggesting that the PPF route was safer than the FO route.

\section{Comparison of efficiency and safety between the foramen rotundum (FR) route and $\mathrm{FO}$ route in $\mathrm{TN}$ treatment via CRF}

Figure 8 shows the comparison of treating effective rate and complication in TN treatment via CRF between the FR group 
Table I Characteristics of the included studies

\begin{tabular}{|c|c|c|c|c|c|c|}
\hline Study ID & $\begin{array}{l}\text { Number of } \\
\text { female (\%)/ } \\
\text { male (\%) } \\
\text { patients }\end{array}$ & Age (years) & $\begin{array}{l}\text { Number of patients } \\
\text { with left }(\%) / \text { right } \\
\text { side pain (\%) }\end{array}$ & $\begin{array}{l}\text { Preoperational } \\
\text { pain (VAS/ } \\
\text { NRS) }\end{array}$ & $\begin{array}{l}\text { Preoperational } \\
\text { pain duration }\end{array}$ & $\begin{array}{l}\text { Preoperational } \\
\text { QoL }\end{array}$ \\
\hline Zhou et al' & $\begin{array}{l}37(6 \mid .7) / 23 \\
(38.3) \\
24(40) / 36(60)\end{array}$ & $\begin{array}{l}54.4 \pm 12.8 \\
61.1 \pm 11.3 \\
55.6 \pm 7.5 \\
61.2 \pm 13.12\end{array}$ & 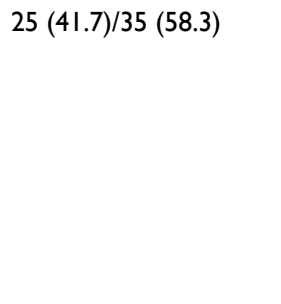 & $\begin{array}{l}\text { NRS: } \\
8.3 \pm 1.0 \\
8.2 \pm 1.8 \\
8.7 \pm 1.0 \\
\text { VAS: } \\
7.32 \pm 1.22 \\
6.99 \pm 1.02 \\
7.22 \pm 1.15\end{array}$ & $\begin{array}{l}72.9 \pm 55.1 \mathrm{~m} \\
90.6 \pm 68.2 \mathrm{~m} \\
63.5 \pm 56.4 \mathrm{~m}\end{array}$ & $\begin{array}{l}32.0 \pm 7.7 \\
28.5 \pm 6.7 \\
27.8 \pm 5.7\end{array}$ \\
\hline $\mathrm{Ma}^{21}$ & $\begin{array}{l}47(52.2) / 43 \\
(47.7)\end{array}$ & $53.6 \pm$ II.2(36-85) & $\begin{array}{l}32(35.5) / 56(62.2) \text {; } \\
\text { bilateral, } 2(2.2)\end{array}$ & & $7 m-20 y$ & \\
\hline Wang et $\mathrm{a}^{22}$ & $18(40) / 27(60)$ & $62(46-78)$ & $12(26.7) / 33(73.3)$ & $\begin{array}{l}\text { VAS: } \\
7.82 \pm 0.56 \\
7.56 \pm 0.75 \\
7.67 \pm 0.77\end{array}$ & 3.2 y $(5 \mathrm{~m}-20 \mathrm{y})$ & \\
\hline $\begin{array}{l}\text { Erdine et } \mathrm{al}^{23} \\
\text { Huibin et a }{ }^{24}\end{array}$ & $\begin{array}{l}21(52.5) / 19 \\
(47.5) \\
28(56) / 22(44)\end{array}$ & $\begin{array}{l}60.05 \pm 11.89 \\
(42-87) \\
64.25 \pm 12.07 \\
(37-85) \\
65.0(38-87)\end{array}$ & $29(58) / 21(42)$ & $\begin{array}{l}\text { VAS: } \\
7-10\end{array}$ & $\begin{array}{l}83.15 \pm 57.8(12-196) \\
\mathrm{m} \\
79.70 \pm 69.9(12-300) \\
\mathrm{m} \\
4.6 \text { y }(1 \mathrm{~m}-30 \mathrm{y})\end{array}$ & \\
\hline Yao et $\mathrm{a}^{25}$ & $\begin{array}{l}31(55.4) / 25 \\
(44.6)\end{array}$ & $\begin{array}{l}55.6 \pm 10.4(32-74) \\
56.1 \pm 12.4(35-75)\end{array}$ & $29(51.8) / 27(48.2)$ & $\begin{array}{l}\text { VAS: } \\
7.6 \pm 1.9 \\
7.7 \pm 1.7\end{array}$ & $\begin{array}{l}9.4 \pm 6.3(5-23) \mathrm{m} \\
10.2 \pm 6.8(4-28) \mathrm{m}\end{array}$ & \\
\hline Yao et $\mathrm{a}^{26}$ & $\begin{array}{l}211(47.3 I) / 235 \\
(52.69) \\
225(51.37) / 213 \\
(48.63) \\
246(52.34) / 224 \\
(47.66)\end{array}$ & $\begin{array}{l}56.35 \pm|3.4|(32- \\
78) ; 61.13 \pm 15.52 \\
(35-82) ; \\
59.24 \pm 14.53 \\
(33-84)\end{array}$ & $\begin{array}{l}587(50.9) / 74 \text { I (54.7); } \\
\text { bilateral, } 26 \text { (I.9) }\end{array}$ & $\begin{array}{l}\text { VAS: } \\
7.22 \pm 2.13 \\
7.46 \pm 1.71 \\
7.29 \pm 2.04\end{array}$ & 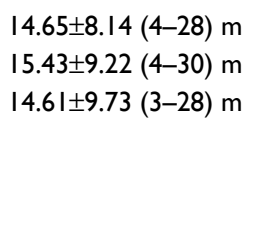 & \\
\hline Elawamy et $\mathrm{al}^{27}$ & $\begin{array}{l}6(50) / 6(50) \\
5(45) / 6(55) \\
13(65) / 7(35)\end{array}$ & $\begin{array}{l}55.75 \pm 11.23 \\
56.00 \pm 10.68 \\
52.60 \pm 9.78\end{array}$ & $\begin{array}{l}40(93) / 3(7) \\
\text { (unilateral/bilateral) }\end{array}$ & $\begin{array}{l}\text { VAS: } \\
8.67 \pm 2.53 \\
9.00 \pm 0.89 \\
9.15 \pm 1.13\end{array}$ & & \\
\hline Kim et $\mathrm{a}^{28}$ & $\begin{array}{l}36(66.7) / 18 \\
(33.3)\end{array}$ & $\begin{array}{l}50.0 \pm 15.9 \\
56.2 \pm 15.8\end{array}$ & $\begin{array}{l}\text { I7 (65.4\%)/6 (23.1\%); } \\
\text { II (39.3\%)/I7 (60.7\%) }\end{array}$ & $\begin{array}{l}\text { VAS: } \\
7.2 \pm 2.0 \\
7.6 \pm 2.0\end{array}$ & & \\
\hline Zhao et $\mathrm{a}^{29}$ & $\begin{array}{l}46(57.5) / 34 \\
(42.5)\end{array}$ & $38-8 \mid(59.3)$ & & & 5.9 y $(0.5-30 y)$ & \\
\hline Yao et $\mathrm{al}^{30}$ & $\begin{array}{l}35(56.5) / 27 \\
(43.5)\end{array}$ & $53.2 \pm$ II.3 (33-78) & & $\begin{array}{l}\text { VAS: } \\
7.5 \pm 1.8\end{array}$ & $10.5 \pm 6.2(4-22) \mathrm{m}$ & \\
\hline Zhai et al ${ }^{15}$ & $\begin{array}{l}22(59.5) / 15 \\
(40.5)\end{array}$ & $60.9 \pm 15.3$ & & $\begin{array}{l}\text { VAS: } \\
10.0(7.0-10.0) \\
9.0(7.0-10.0) \\
10.0(7.0-10.0)\end{array}$ & & \\
\hline
\end{tabular}




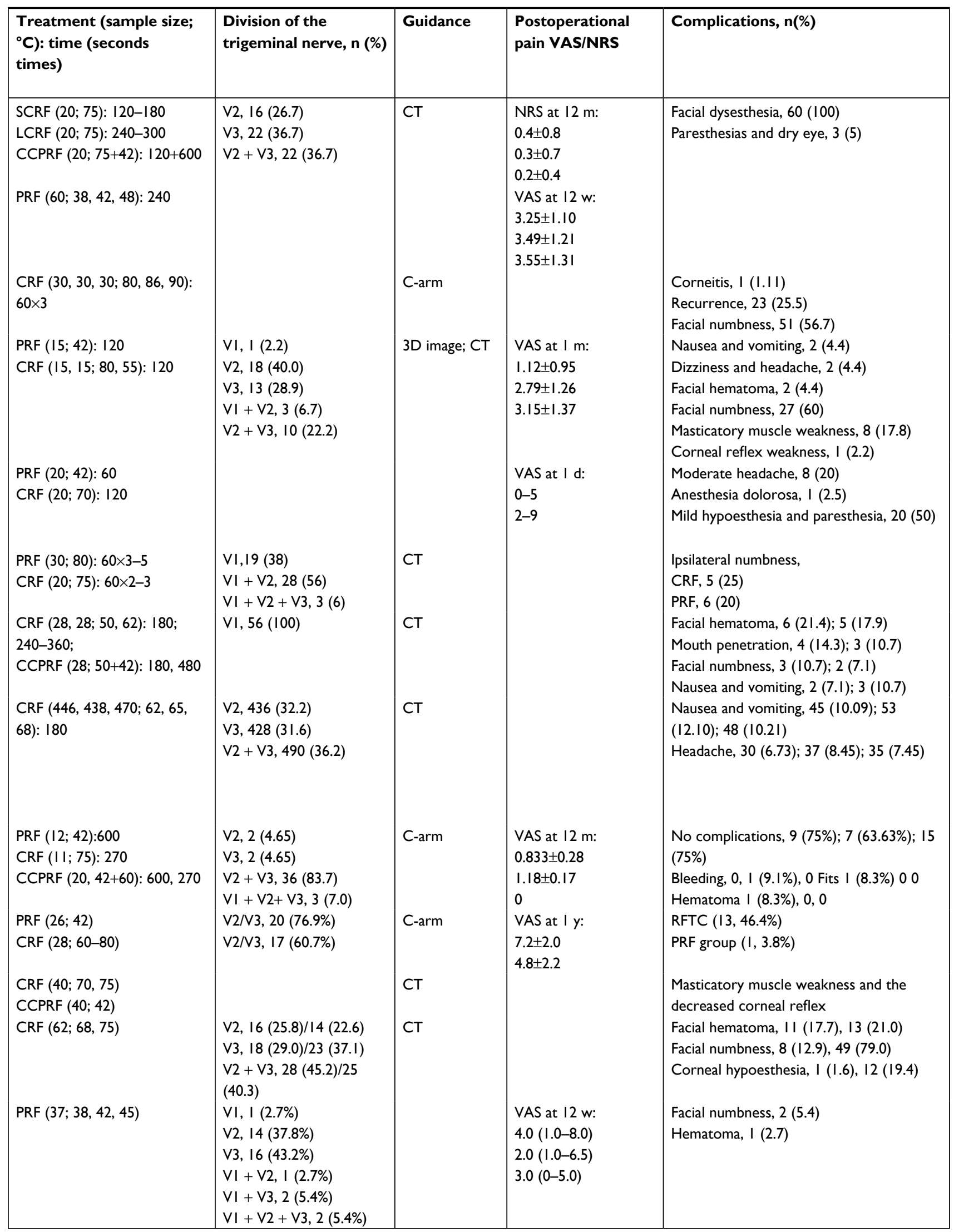

(Continued) 
Table I Continued

\begin{tabular}{|c|c|c|c|c|c|c|}
\hline Study ID & $\begin{array}{l}\text { Number of } \\
\text { female (\%)/ } \\
\text { male (\%) } \\
\text { patients }\end{array}$ & Age (years) & $\begin{array}{l}\text { Number of patients } \\
\text { with left (\%)/right } \\
\text { side pain (\%) }\end{array}$ & $\begin{array}{l}\text { Preoperational } \\
\text { pain (VAS/ } \\
\text { NRS) }\end{array}$ & $\begin{array}{l}\text { Preoperational } \\
\text { pain duration }\end{array}$ & $\begin{array}{l}\text { Preoperational } \\
\text { QoL }\end{array}$ \\
\hline Hu and Wang ${ }^{32}$ & $\begin{array}{l}53(58.9) / 37 \\
(41.1) \\
35(58.3) / 25 \\
(41.7)\end{array}$ & $\begin{array}{l}\text { CRF: } 62.1 \pm 10.2 \\
\text { CRF + PRF: } \\
60.9 \pm 10.4 \\
\text { PRF: } 67.2(43-76) \\
\text { CRF: } 68.7(56-75)\end{array}$ & $23(38.3) / 37(61.7)$ & $\begin{array}{l}\text { VAS: } \\
7.90 \pm 1.37 \\
8.18 \pm 1.34\end{array}$ & $\begin{array}{l}94.4 \pm 66.5 \mathrm{~m} \\
95.2 \pm 97.7 \mathrm{~m} \\
2-13 y \\
1.5-14 y\end{array}$ & $\begin{array}{l}28.9 \pm 6.11 \\
28.0 \pm 6.25\end{array}$ \\
\hline Meng et $\mathrm{al}^{33}$ & $48(48) / 52(52)$ & $\begin{array}{l}\text { PRF: } 66.18 \pm I I .66 \\
\text { CRF: } 63.29 \pm 8.87\end{array}$ & $43(43) / 57(57)$ & $\begin{array}{l}\text { NRS: } 7.18 \pm 1.69 \\
7.11 \pm 1.82\end{array}$ & & \\
\hline Han et $\mathrm{al}^{34}$ & $\begin{array}{l}17(39.5) / 26 \\
(60.5)\end{array}$ & $\begin{array}{l}\text { 3D: } \\
68.1 \pm 10.1 \\
C T: \\
68.6 \pm 11.1\end{array}$ & & $\begin{array}{l}\text { NRS: } 3.1 \pm I .7 \\
3.1 \pm I .5\end{array}$ & $\begin{array}{l}6.3 \pm 3.8 y \\
5.9 \pm 3.6 y\end{array}$ & \\
\hline Lu et $\mathrm{al}^{35}$ & $36 / 24$ & $\begin{array}{l}\text { 3D: } \\
61.9 \pm 12.1 \\
C T: \\
62.4 \pm 11.9\end{array}$ & & $\begin{array}{l}\text { NRS: } \\
9 \\
9\end{array}$ & $\begin{array}{l}5.4 \pm 2.7 y \\
5.1 \pm 3.1 y\end{array}$ & \\
\hline Nie et $\mathrm{a}^{36}$ & $36(60) / 24(40)$ & $62(42-84)$ & 3 I (5I.7)/29 (48.3) & $\begin{array}{l}\text { VAS: } \\
8.1 \pm 1.92 \\
8.0 \pm 1.94\end{array}$ & $\begin{array}{l}24 \pm 18.3 \mathrm{~m} \\
28 \pm 20.5 \mathrm{~m}\end{array}$ & \\
\hline $\begin{array}{l}\text { Nie et } \mathrm{al}^{37} \\
\text { Qu et } \mathrm{a}^{38}\end{array}$ & & $\begin{array}{l}58 \pm 6.8 \\
60 \pm 9.6 \\
57 \pm 7.2\end{array}$ & & $\begin{array}{l}\text { VAS: } \\
8.9 \pm 0.92 \\
9.0 \pm 0.78 \\
8.7 \pm 0.94\end{array}$ & $\begin{array}{l}28 \pm 18.3 \mathrm{~m} \\
32 \pm 16.8 \mathrm{~m} \\
27 \pm 20.5 \mathrm{~m}\end{array}$ & \\
\hline Yang et al ${ }^{39}$ & $\begin{array}{l}75(59.1) / 52 \\
(40.9)\end{array}$ & $57.0(38-64)$ & $44(34.6) / 83$ (65.4) & $\begin{array}{l}\text { VAS: } \\
9.2 \pm 0.4 \\
9.1 \pm 0.5\end{array}$ & $\begin{array}{l}17.1 \pm 5.8 \mathrm{~m} \\
15.2 \pm 6.7 \mathrm{~m}\end{array}$ & \\
\hline Chang et $\mathrm{a}^{40}$ & $\begin{array}{l}107(66.9) / 53 \\
(33.1)\end{array}$ & $66(37-94)$ & $\begin{array}{l}62(38.75) / 95(59.4) \\
\text { bilateral, } 3(1.9)\end{array}$ & & $67.2(1-360) \mathrm{m}$ & \\
\hline Wang et $\mathrm{a}^{41}$ & $\begin{array}{l}49(6 \mid .25) / 31 \\
(38.75)\end{array}$ & $\begin{array}{l}71.1 \pm 2.6(60-80) \\
71.2 \pm 2.5(60-80)\end{array}$ & $33(4 \mathrm{I} .25) / 47$ (58.75) & $\begin{array}{l}\text { VAS: } \\
7.2 \pm 0.3 \\
7.2 \pm 0.4\end{array}$ & $\begin{array}{l}37.2 \pm 2.4(I-96) \mathrm{m} \\
38.4 \pm 2.4(\mathrm{I}-96) \mathrm{m}\end{array}$ & \\
\hline Fu et $\mathrm{al}^{42}$ & $\begin{array}{l}28(33.3) / 56 \\
(66.7)\end{array}$ & $56 \pm 5(4 \mid-68)$ & & & & \\
\hline Cao et $\mathrm{al}^{43}$ & $\begin{array}{l}58(64.4) / 32 \\
(35.6)\end{array}$ & $\begin{array}{l}53.69 \pm 12.45 \\
53.38 \pm 12.02\end{array}$ & & $\begin{array}{l}\text { VAS: } \\
8.27 \pm 1.02 \\
8.20 \pm 1.08\end{array}$ & & \\
\hline
\end{tabular}




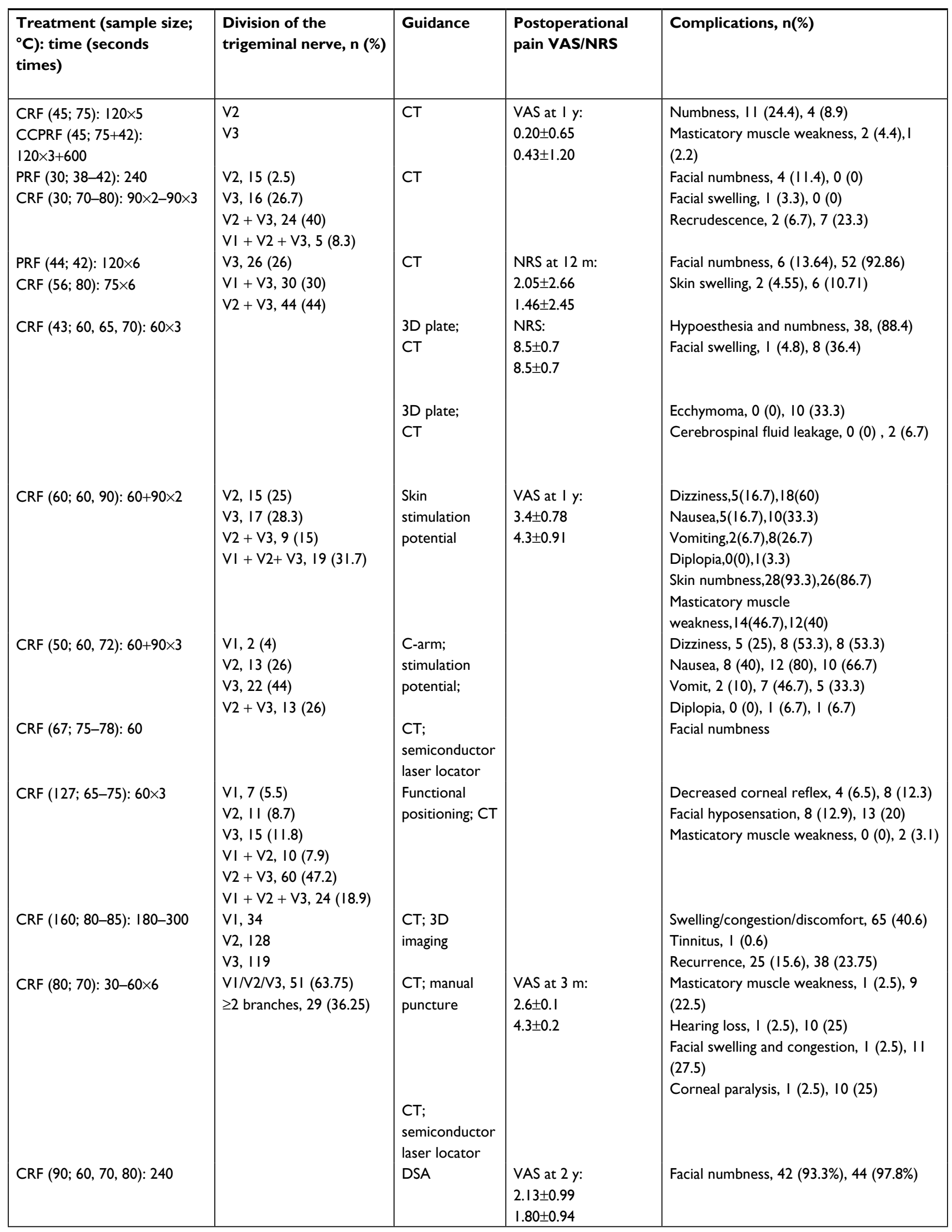

(Continued) 
Table I Continued

\begin{tabular}{|c|c|c|c|c|c|c|}
\hline Study ID & $\begin{array}{l}\text { Number of } \\
\text { female (\%)/ } \\
\text { male (\%) } \\
\text { patients }\end{array}$ & Age (years) & $\begin{array}{l}\text { Number of patients } \\
\text { with left }(\%) / \text { right } \\
\text { side pain (\%) }\end{array}$ & $\begin{array}{l}\text { Preoperational } \\
\text { pain (VAS/ } \\
\text { NRS) }\end{array}$ & $\begin{array}{l}\text { Preoperational } \\
\text { pain duration }\end{array}$ & $\begin{array}{l}\text { Preoperational } \\
\text { QoL }\end{array}$ \\
\hline Huang et al ${ }^{44}$ & $\begin{array}{l}24(60 \%) / 16 \\
(40 \%)\end{array}$ & $68.1(52-78)$ & $13(32.5) / 27(67.5)$ & & 5 y $(4 \mathrm{~m}-14 \mathrm{y})$ & \\
\hline Chen et $\mathrm{a}^{45}$ & $\begin{array}{l}45(65.2) / 24 \\
(34.8)\end{array}$ & $\begin{array}{l}70.33(43-87) \\
67.39(30-83)\end{array}$ & & & $>1$ y & \\
\hline Jiang et $\mathrm{al}^{46}$ & $31(62) / 19$ (38) & $63 \pm 25(36-86)$ & & $\begin{array}{l}\text { VAS: } \\
8.2 \pm 1.0 \\
7.9 \pm 1.5\end{array}$ & $5 m-18 y$ & \\
\hline Liao et $\mathrm{al}^{47}$ & $\begin{array}{l}16(38.1) / 26 \\
(69.1)\end{array}$ & $\begin{array}{l}72.2 \pm 2.4 \\
74.4 \pm 3.4\end{array}$ & & $\begin{array}{l}\text { VAS: } \\
8.4 \pm 0.9 \\
8.6 \pm 0.7\end{array}$ & $\begin{array}{l}3.3 \pm 0.7 y \\
4.5 \pm 0.8 y\end{array}$ & $\begin{array}{l}26 \pm 5 \\
26 \pm 4\end{array}$ \\
\hline Yao et $\mathrm{a}^{48}$ & $\begin{array}{l}19(55.88) / 15 \\
(44.12)\end{array}$ & $\begin{array}{l}55.8 I \pm 15.33 \\
(30-74)\end{array}$ & & & $7.53 \pm 5.24(4-18) \mathrm{m}$ & \\
\hline $\begin{array}{l}\text { Zhao and } \\
\text { Song }{ }^{49}\end{array}$ & $\begin{array}{l}34(56.7) / 26 \\
(43.3)\end{array}$ & $62.5(42-80)$ & & $\begin{array}{l}\text { VAS: } \\
8.87 \pm 0.86 \\
8.80 \pm 0.85\end{array}$ & $3 \mathrm{~m}-10 \mathrm{y}$ & \\
\hline Jiang et $\mathrm{al}^{50}$ & $\begin{array}{l}43(53.75) / 37 \\
(46.25)\end{array}$ & $\begin{array}{l}65.7 \pm 8.5 \\
66.6 \pm 8.7\end{array}$ & $32(40) / 48(60)$ & & 2.3 y $(5 \mathrm{~m}-10 \mathrm{y})$ & \\
\hline Wu et $a^{\mid 51}$ & $\begin{array}{l}28(34.6) / 53 \\
(65.4)\end{array}$ & 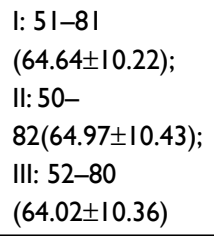 & $4 \mathrm{I}(50.6) / 40$ (49.4) & & $\begin{array}{l}\text { I: } 4 \mathrm{~m}-9 \text { y } \\
(47.95 \pm 7.58) \mathrm{m} ; \\
\text { II: } 5 \mathrm{~m}-8 \mathrm{y} \\
(47.06 \pm 7.95) \mathrm{m} \\
\text { III: } 6 \mathrm{~m}-9 \mathrm{y} \\
(47.12 \pm 7.04) \mathrm{m}\end{array}$ & \\
\hline
\end{tabular}

Notes: Data described as mean \pm SD or mean (range) (except for the VAS score in Zhai et al ${ }^{15}$ which was described as M(P25, P75)), VI-V3: branches of cranial nerve $\mathrm{V}$ (the trigeminal nerve). Those items could not be extracted from the original articles are left empty.

Abbreviations: 3D, three-dimensional; CCPRF, pulsed radiofrequency + continuous radiofrequency group; CRF, continuous radiofrequency group; CT, computed tomography; d, day; DSA, digital subtraction angiography; LCRF, long-time CRF; m, month; NRS, numerical rating scale; PRF, pulsed radiofrequency group; QoL, quality of life; RFTC, conventional radiofrequency thermocoagulation; SCRF, short-time CRF; VAS, visual analog scale; w, week; $y$, year.

and FO group. Three studies ${ }^{29,44,45}$ in the FR group compared with FO study showed acceptable heterogeneity of the studies $\left(\chi^{2}=2.97, P=0.23>0.05, \mathrm{I}^{2}=33 \%\right)$; the total effect size OR in this study was 2.86 (95\% CI: $0.75,10.96)$, and the $Z$ value was $1.53(P=0.13)$, indicating that $F R$ and $F O$ were not associated with an effective rate of TN treatment. Three studies $^{29,44,45}$ in FR compared with FO patients showed less heterogeneity of the studies $\left(\chi^{2}=2.28, P=0.32>0.05, \mathrm{I}^{2}=12 \%\right)$; the total effect size OR in this study was 0.01 (95\% CI: 0.00 , $0.07)$, and the $Z$ value was $4.92(P<0.00001)$, indicating that the FR route was safer.

\section{Comparison of efficiency between different temperatures in TN treatment via PRF}

Figure 9 shows the effect of different temperatures of PRF thermocoagulation on TN treatment. Three studies ${ }^{14,15,50}$ in the low-temperature group $\left(38^{\circ} \mathrm{C}-44^{\circ} \mathrm{C}\right)$ compared with the high-temperature group $\left(45^{\circ} \mathrm{C}-50^{\circ} \mathrm{C}\right)$ patients showed less heterogeneity of the studies $\left(\chi^{2}=3.14, P=0.21>0.05, \mathrm{I}^{2}=36 \%\right)$; the total effect size OR in this study was 0.32 (95\% CI: 0.14 , $0.73)$, and the $\mathrm{Z}$ value was $2.71(P=0.007<0.05)$, indicating that the higher temperature group $\left(45^{\circ} \mathrm{C}-50^{\circ} \mathrm{C}\right)$ has a greater effect on the treatment.

\section{Subgroup analysis of efficiency and safety between different temperatures in TN treatment via CRF}

Figure 10 shows the comparison of the effect and complication of different temperatures $\left(68^{\circ} \mathrm{C}-70^{\circ} \mathrm{Cvs} 71^{\circ} \mathrm{C}-75^{\circ} \mathrm{C}\right)$ in $\mathrm{TN}$ treatment. Two studies ${ }^{30,48}$ in the lower temperature group $\left(68^{\circ} \mathrm{C}-70^{\circ} \mathrm{C}\right)$ compared with the higher temperature group $\left(71^{\circ} \mathrm{C}-75^{\circ} \mathrm{C}\right)$ patients showed no heterogeneity of the studies (both $P>0.05, \mathrm{I}^{2}=0 \%$ ); the total effect size OR in this study was 1.79 (95\% CI: 0.94, 3.42), and the $Z$ value was $1.77(P=0.08>0.05)$, suggesting that there was no signifi- 


\begin{tabular}{|c|c|c|c|c|}
\hline $\begin{array}{l}\text { Treatment (sample size; } \\
\left.{ }^{\circ} \mathrm{C}\right) \text { : time (seconds } \\
\text { times) }\end{array}$ & $\begin{array}{l}\text { Division of the } \\
\text { trigeminal nerve, } n(\%)\end{array}$ & Guidance & $\begin{array}{l}\text { Postoperational } \\
\text { pain VAS/NRS }\end{array}$ & Complications, n(\%) \\
\hline $\operatorname{CRF}(40 ; 60,65,70,75): 60 \times 4$ & V2, $40(100 \%)$ & CT & & Branch injury of trigeminal nerve, 17 (85\%) \\
\hline $\operatorname{CRF}(69 ; 60-84): 240-300$ & & CT & & V3 hypoesthesia, 25 (75.76\%) \\
\hline $\operatorname{CRF}(50 ; 60,70,80): 240$ & V2, $50(100 \%)$ & DSA & $\begin{array}{l}\text { VAS: } \\
3.9 \pm 1.3 \\
4.2 \pm 1.2\end{array}$ & $\begin{array}{l}\text { Branch injury of trigeminal nerve, VI, I } \\
(2 \%) \text {; V3, } 3(6 \%)\end{array}$ \\
\hline CRF $(42 ; 60,70,75,80): 360$ & V2, $42(100 \%)$ & DSA & $\begin{array}{l}\text { VAS at I } y: \\
1.2 \pm 0.2 \\
1.2 \pm 0.2\end{array}$ & $\begin{array}{l}\text { Corneal reflexes, I }(4.5 \%) \\
\text { Masticatory atonia, } 2(9.1 \%) \\
\text { Numbness, } 6(27.3 \%)\end{array}$ \\
\hline CRF $(34 ; 70,75)$ & $\begin{array}{l}V 2,18 \\
V 3,20 \\
V 2+V 3,30\end{array}$ & CT & $\begin{array}{l}\text { NRS: } \\
7.6 I \pm I .73\end{array}$ & $\begin{array}{l}\text { Facial numbness, } 5 \text { (14.7), } 27 \text { (79.4) } \\
\text { Masticatory atonia, } 2(5.9), \text { I } 5 \text { (44.I) } \\
\text { Corneal reflex weakness, I (2.9), } 9 \text { (26.5) }\end{array}$ \\
\hline CRF $(60 ; 72): 75 \times 3$ & & CT & $\begin{array}{l}\text { VAS: } \\
\text { I. } 17 \pm 0.87 \\
I .27 \pm 0.74\end{array}$ & $\begin{array}{l}\text { Trigeminal nerve injury, VI, I (3.3\%); V3, } \\
4 \text { (I3.3\%), } \\
\text { Nausea and vomiting, } 10 \text { (33.3\%) }\end{array}$ \\
\hline PRF $(80 ; 42,50): 120 \times 4$ & & C-arm & & \\
\hline CRF (8I; 80, 85, 90): 90 & & $\mathrm{CT}$ & & $\begin{array}{l}\text { Facial numbness } \\
\text { I IO (37.04\%) } \\
\text { II } 4(14.81 \%) \\
\text { III II (40.74\%) }\end{array}$ \\
\hline
\end{tabular}

cance of the effect of different temperatures $\left(68^{\circ} \mathrm{C}-70^{\circ} \mathrm{C}\right.$ vs $71^{\circ} \mathrm{C}-75^{\circ} \mathrm{C}$ ) of CRF in TN treatment. The total effect size OR in this study was 0.04 (95\% CI: 0.02, 0.09), and the $Z$ value was $8.17(P<0.00001)$, indicating that lower temperature therapy $\left(68^{\circ} \mathrm{C}-70^{\circ} \mathrm{C}\right)$ was safer.

Figure 11 shows the effect and complications of different temperatures $\left(66^{\circ} \mathrm{C}-80^{\circ} \mathrm{C}\right.$ vs $\left.55^{\circ} \mathrm{C}-65^{\circ} \mathrm{C}\right)$. Two studies ${ }^{22,26}$ in the higher temperature group $\left(66^{\circ} \mathrm{C}-80^{\circ} \mathrm{C}\right)$ compared with lower temperature group $\left(55^{\circ} \mathrm{C}-65^{\circ} \mathrm{C}\right)$ patients showed no heterogeneity of the studies $\left(\chi^{2}=0.64, P=0.42>0.05, \mathrm{I}^{2}=0\right.$ $\%)$. The total effect size OR in this study was $2.77(95 \%$ CI: $2.02,3.80)$, and the $\mathrm{Z}$ value was $6.33(P<0.00001)$. Two studies $^{22,26}\left(66^{\circ} \mathrm{C}-80^{\circ} \mathrm{C}\right.$ vs $\left.55^{\circ} \mathrm{C}-65^{\circ} \mathrm{C}\right)$ showed no heterogeneity $\left(\chi^{2}=0.17, P=0.68>0.05, \mathrm{I}^{2}=0 \%\right)$; the total effect size OR in this study was 4.58 (95\% CI: $3.21,6.54)$, and the $\mathrm{Z}$ value was $8.40(P<0.00001)$, indicating that relatively higher temperature group $\left(66^{\circ} \mathrm{C}-80^{\circ} \mathrm{C}\right)$ has a greater effect on $\mathrm{TN}$ treatment, while the safety of which is decreasing.

Figure 12 shows the effect and complications of different temperatures $\left(80^{\circ} \mathrm{C}-85^{\circ} \mathrm{C}\right.$ vs $\left.86^{\circ} \mathrm{C}-90^{\circ} \mathrm{C}\right)$. Two studies ${ }^{21,51}$ in the lower temperature group $\left(80^{\circ} \mathrm{C}-85^{\circ} \mathrm{C}\right)$ compared with higher temperature group $\left(86^{\circ} \mathrm{C}-90^{\circ} \mathrm{C}\right)$ patients showed no heterogeneity of the studies $\left(\chi^{2}=0.01, P=0.94>0.05, \mathrm{I}^{2}=0 \%\right)$; the total effect size OR in this study was 0.91 (95\% CI: 0.43 , $1.93)$, and the $Z$ value was $0.26(P=0.80>0.05)$. Two studies $^{21,51}\left(80^{\circ} \mathrm{C}-85^{\circ} \mathrm{C}\right.$ vs $\left.86^{\circ} \mathrm{C}-90^{\circ} \mathrm{C}\right)$ showed no heterogeneity $\left(\chi^{2}=0.49, P=0.48>0.05, \mathrm{I}^{2}=0 \%\right)$; the total effect size OR in this study was 0.66 (95\% CI: $0.34,1.28)$, and the $Z$ value was $1.23(P=0.22>0.05)$, indicating that the change in temperature $>80^{\circ} \mathrm{C}$ had no significant difference in the efficiency and safety during the $\mathrm{TN}$ treatment.

\section{Discussion}

$\mathrm{TN}$ is a clinically common painful disease, and a variety of drugs or surgical procedures are available for its treatment. Medications such as carbamazepine oxcarbazepine, baclofen, lamotrigine, phenytoin, and topiramate could be administered to control pain. Intravenous infusion of a combination of magnesium and lidocaine can be very effective in some patients. However, different extents of side effects could occur after drug treatment. Procedures such as radiosurgery, percutaneous balloon compression, glycerol rhizotomy, radiofrequency thermocoagulation, peripheral nerve dissection, partial sensory nerve root dissection, and 
Table 2 Methodological quality assessment of the included studies via modified Jadad scale

\begin{tabular}{|c|c|c|c|c|c|}
\hline Included study & $\begin{array}{l}\text { Random sequence } \\
\text { production }\end{array}$ & $\begin{array}{l}\text { Allocation } \\
\text { concealment }\end{array}$ & Blind method & Withdraw/quit & Total score \\
\hline $\mathrm{Li}$ et $\mathrm{al}^{20}$ & 2 & 2 & I & I & 6 \\
\hline Zhou et al ${ }^{14}$ & 0 & 0 & 0 & I & 1 \\
\hline $\mathrm{Ma}^{21}$ & 0 & 0 & 0 & 0 & 0 \\
\hline Wang et $\mathrm{a}^{22}$ & 2 & I & 0 & 0 & 3 \\
\hline Edrine et $\mathrm{al}^{23}$ & 2 & 2 & I & 0 & 5 \\
\hline Qin et $\mathrm{a}^{24}$ & 2 & 1 & 0 & 0 & 3 \\
\hline Yao et $\mathrm{al}^{25}$ & 2 & I & I & 0 & 4 \\
\hline Yao et $\mathrm{al}^{26}$ & 2 & I & I & I & 5 \\
\hline Elawamy et $\mathrm{al}^{27}$ & 2 & 2 & I & I & 6 \\
\hline Kim et $\mathrm{al}^{28}$ & I & 0 & 0 & 0 & I \\
\hline Zhao et $\mathrm{al}^{29}$ & I & 0 & 0 & I & 2 \\
\hline Yao et $\mathrm{al}^{30}$ & I & 0 & 0 & I & 2 \\
\hline Zhai et al ${ }^{15}$ & 2 & I & 0 & I & 4 \\
\hline Li et $\mathrm{al}^{31}$ & 2 & I & 0 & 0 & 3 \\
\hline $\mathrm{Hu}$ and Wang $\mathrm{W}^{32}$ & I & 0 & 0 & 0 & I \\
\hline Meng et $\mathrm{al}^{33}$ & I & 0 & 0 & 0 & I \\
\hline Han et $\mathrm{al}^{34}$ & 2 & 1 & 0 & 0 & 3 \\
\hline Lu et $\mathrm{a}^{35}$ & 2 & I & 0 & 0 & 3 \\
\hline $\mathrm{Nie}$ et $\mathrm{al}^{36}$ & I & 0 & 0 & 0 & I \\
\hline Nie et $\mathrm{al}^{37}$ & 0 & 0 & 0 & 0 & 0 \\
\hline Qu et $\mathrm{al}^{38}$ & 2 & I & 0 & 0 & 3 \\
\hline Yang et al ${ }^{19}$ & I & 0 & 0 & 0 & I \\
\hline Chang et $\mathrm{al}^{40}$ & I & 0 & 0 & 0 & I \\
\hline Wang et $\mathrm{al}^{41}$ & 2 & I & 0 & 0 & 3 \\
\hline Fu et $\mathrm{al}^{42}$ & I & 0 & 0 & 0 & I \\
\hline Cao et $\mathrm{al}^{43}$ & I & 0 & 0 & I & 2 \\
\hline Huang et $\mathrm{a}^{44}$ & 0 & 0 & 0 & I & I \\
\hline Chen et $\mathrm{a}^{45}$ & I & 0 & 0 & I & 2 \\
\hline Jiang et $\mathrm{al}^{46}$ & I & 0 & 0 & I & 2 \\
\hline Liao et $\mathrm{al}^{47}$ & I & 0 & 0 & I & 2 \\
\hline Yao et $\mathrm{al}^{48}$ & I & I & I & I & 4 \\
\hline Zhao and Song ${ }^{49}$ & I & 0 & 0 & I & 2 \\
\hline Jiang et $\mathrm{al}^{50}$ & I & 0 & 0 & I & 2 \\
\hline Wu et $a^{51}$ & 2 & I & 0 & 0 & 3 \\
\hline
\end{tabular}

microvascular decompression could be utilized. Similarly, partial sensory nerve root dissection and microvascular decompression may not have persistent curative effect, while peripheral nerve dissection could lead to a loss of partial facial sensation. ${ }^{52}$

$\mathrm{CRF}$, as a less invasive and effective treatment, has been widely applied to the treatment of TN patients who are refractory to medical therapy since $1974 .{ }^{53}$ The heat produced by the radiofrequency needle is thought to selectively destroy the $\mathrm{A}$ and $\mathrm{C}$ pain fibers by thermocoagulation at temperatures $>65^{\circ} \mathrm{C}$, while the medullary fibers (A fibers) that conduct the tactile sensation can tolerate higher temperature. Some results showed that there was a significant difference in the efficacy of PRF with less postoperative complications, but the recurrence time was shorter than that of $\mathrm{CRF}^{22} \mathrm{PRF}$ achieves an analgesic purpose by stimulating the nerve instead of damaging it; therefore, the effect duration turns to be shorter. ${ }^{33,54}$ The idea of combined application of CRF and PRF, sometimes named as CCPRF, could not only reduce the excessive damage of the CRF to the nerve tissue but also decrease the occurrence of the complications to some extent. Although PRF and CRF had no statistical difference in cured rate, CCPRF had a greater effect on treatment than CRF, while PRF and CCPRF had no difference. ${ }^{27}$ Furthermore, some studies suggested that its long-term efficacy was not as good as the simple CRF. ${ }^{31}$ The advantage of CCPRF is still in need of further investigation.

As our results showed, there is a big lift in the success rate at first puncture when combining extra guidance techniques such as semiconductor laser locator and stimulation potential guidance other than simple image guidance approach. Interestingly, the recent 3D-printed template guidance achieved a greater success rate at first puncture than that of simple image guidance approach, indicating that 3D-printed template might 


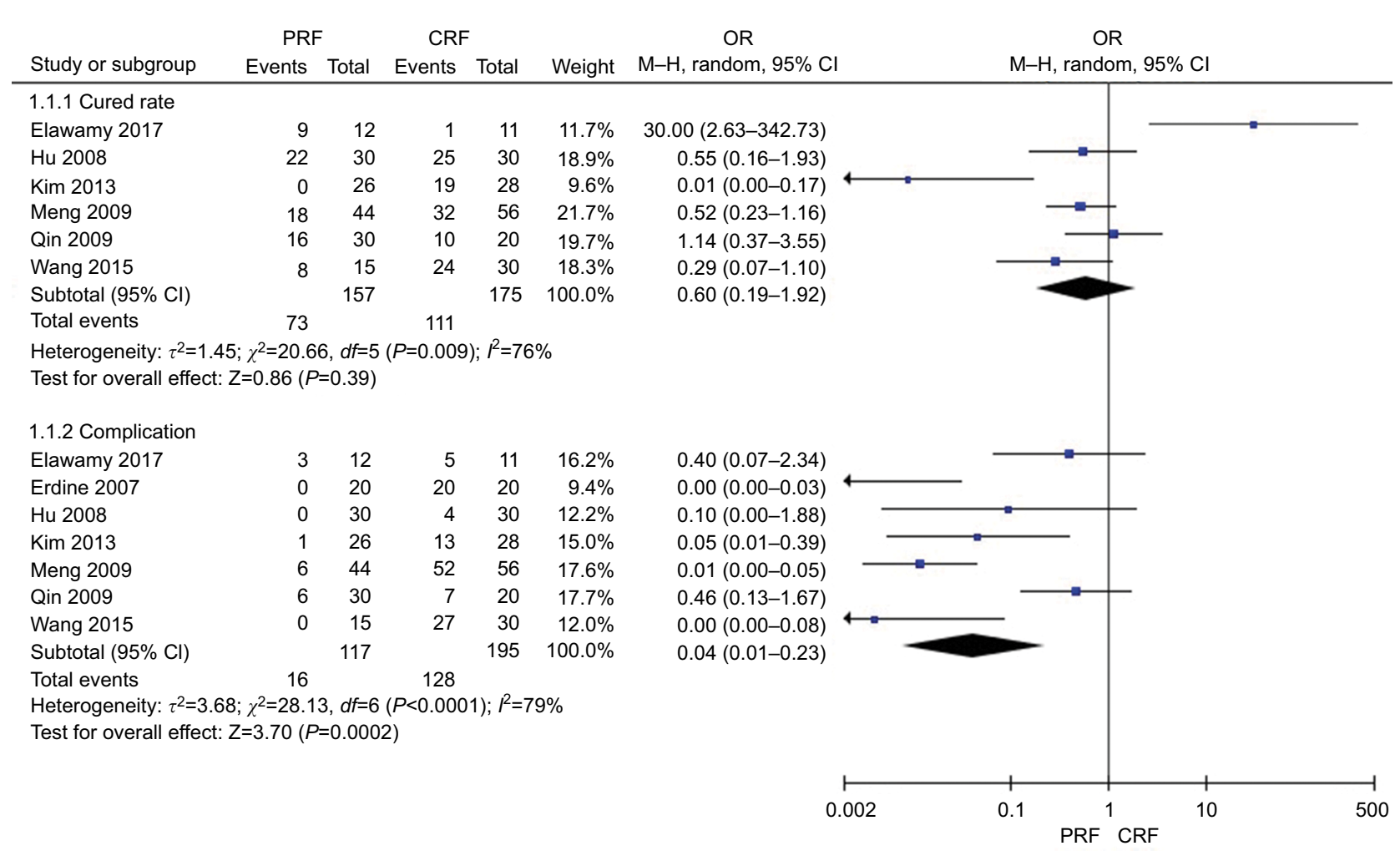

Figure 2 Comparison of the effect and safety of PRF and CRF in the treatment of TN patients.

Abbreviations: CRF, continuous radiofrequency; M-H, Mantel-Haenszel method; PRF, pulsed radiofrequency group; TN, trigeminal neuralgia.

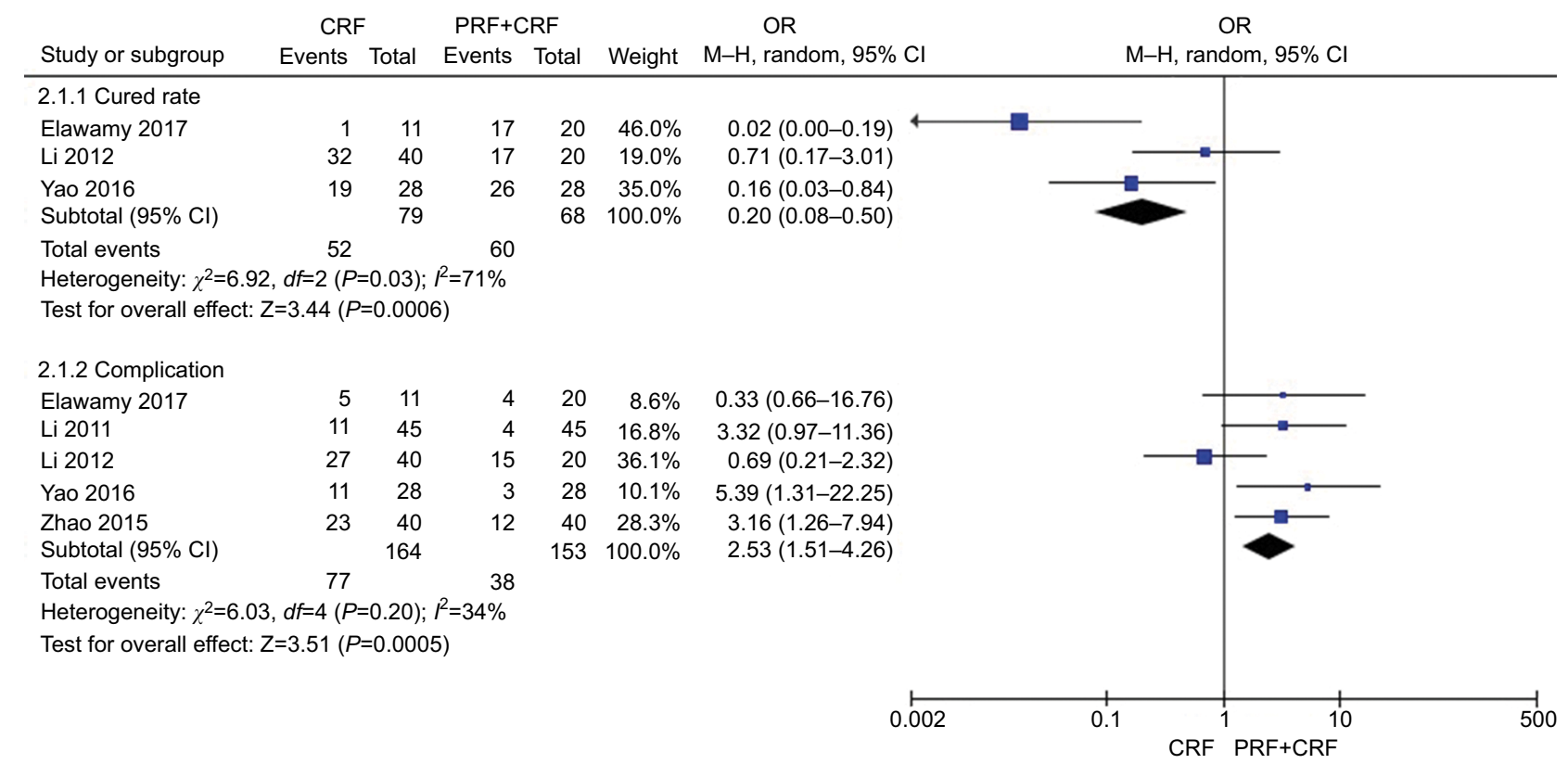

Figure 3 Comparison of the effect and safety of CRF and CCPRF in the treatment of TN patients.

Abbreviations: CCPRF, pulsed radiofrequency + continuous radiofrequency group; CRF, continuous radiofrequency; PRF, pulsed radiofrequency group; TN, trigeminal neuralgia.

be a potential guidance for $\mathrm{TN}$ treatment. We thought that 3D-printed template might provide benefits for making an evaluation and the best puncture plan, including puncture point, angle, and depth. Traditional puncturing methods rely on the experience of the surgeon and the recognition of the anatomical structure near the FO mostly, and it is sometimes in need of repetitive punctures due to the individual differences, while 3D-printed template can avoid this. 


\begin{tabular}{|c|c|c|c|c|c|c|c|c|}
\hline \multirow[b]{2}{*}{ Study or subgroup } & \multicolumn{2}{|c|}{$3 \mathrm{D} C \mathrm{CT}$} & \multicolumn{2}{|c|}{ Manual puncture } & \multicolumn{2}{|r|}{ OR } & \multirow{2}{*}{$\begin{array}{c}\text { OR } \\
\mathrm{M}-\mathrm{H}, \text { fixed, } 95 \% \mathrm{Cl}\end{array}$} & \\
\hline & Events & Total & Events & Total & Weight & $\mathrm{M}-\mathrm{H}$, fixed, $95 \% \mathrm{Cl}$ & & \\
\hline Chang 2008 & 54 & 80 & 62 & 80 & $74.6 \%$ & $0.60(0.30-1.22)$ & & \\
\hline Wang 2017 & 15 & 40 & 11 & 40 & $25.4 \%$ & $1.58(0.62-4.07)$ & & \\
\hline Total $(95 \% \mathrm{Cl})$ & & 120 & & 120 & $100.0 \%$ & $0.85(0.49-1.48)$ & & \\
\hline Total events & 69 & & 73 & & & & & \\
\hline $\begin{array}{l}\text { Heterogeneity: } \chi^{2}= \\
\text { Test for overall effe }\end{array}$ & $\begin{array}{l}d f=1(P= \\
=0.57(P=\end{array}$ & $\begin{array}{l}0.11) ; P^{2}= \\
0.57)\end{array}$ & $61 \%$ & & & 0.01 & $0.13 \mathrm{D} \mathrm{CT}^{1}$ Manual $\mathrm{p}$ & $\begin{array}{l}10 \\
\text { uncture }\end{array}$ \\
\hline
\end{tabular}

Figure 4 Comparison of the effect of guidance (3D CT vs manual puncture) in CRF treatment.

Abbreviations: CRF, continuous radiofrequency; CT, computed tomography; 3D, three-dimensional.

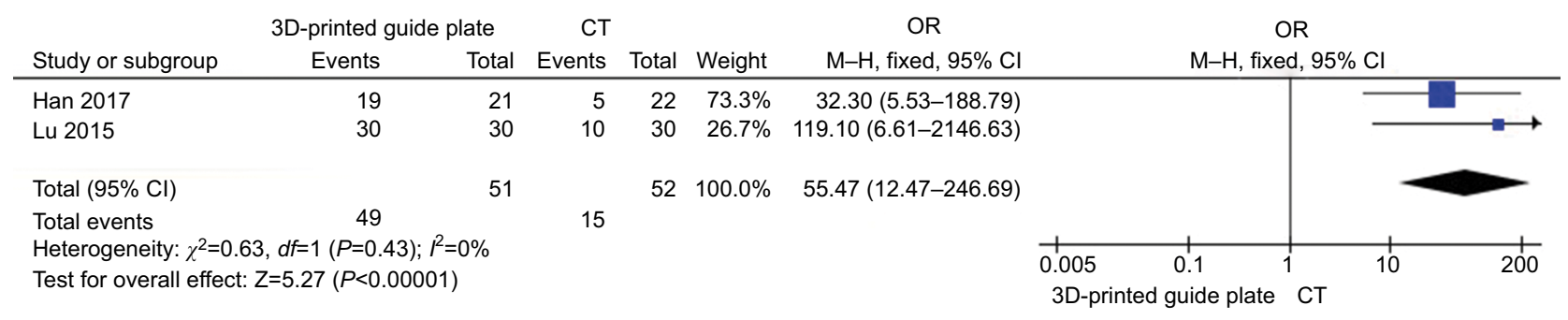

Figure 5 Comparison of the effect of guidance (3D-printed guide plate vs CT guide) in CRF treatment. Abbreviations: CRF, continuous radiofrequency; CT, computed tomography; 3D, three-dimensional.

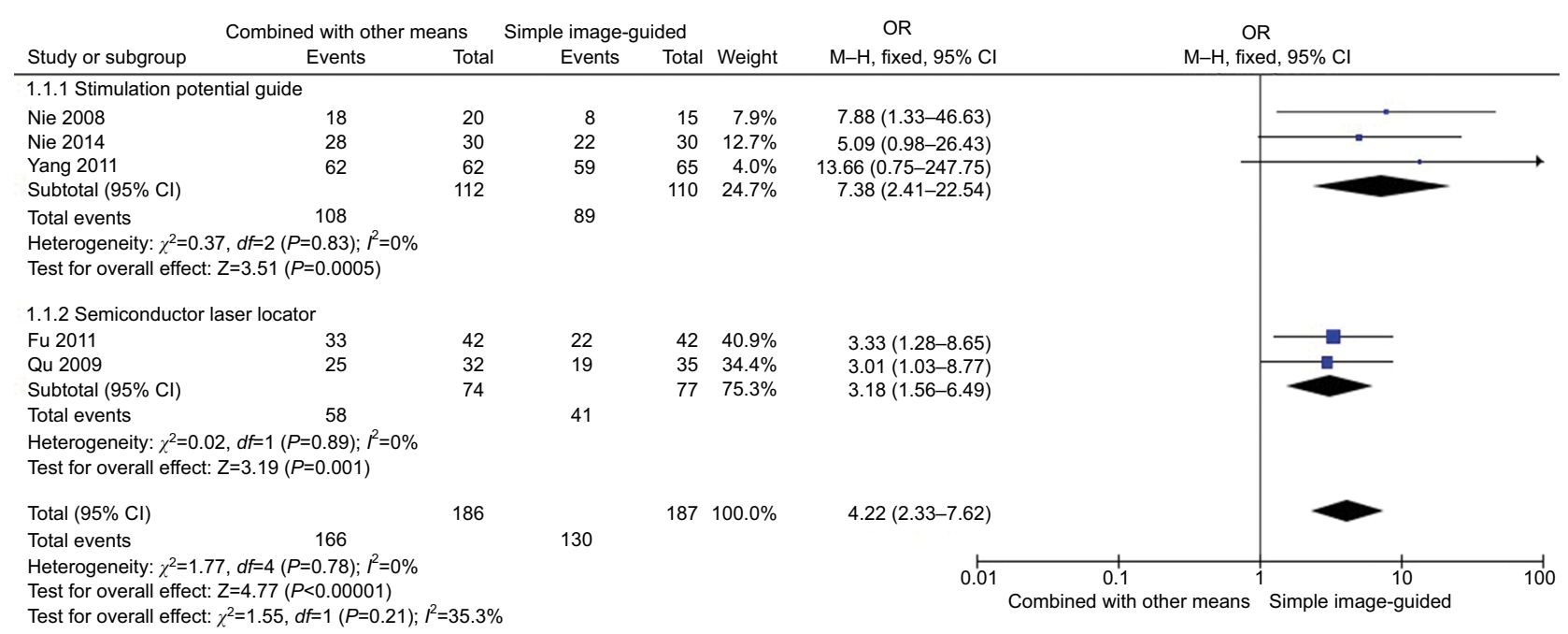

Figure 6 Comparison of the effect of different guidance (combined with other means vs simple image guidance) in CRF treatment. Abbreviation: CRF, continuous radiofrequency.

At present, the selection of CRF temperatures in TN treatment has no specific standards. Various studies used temperatures ranging from $55^{\circ} \mathrm{C}$ to $90^{\circ} \mathrm{C}$. Temperature $>65^{\circ} \mathrm{C}$ was known to destroy nerve fibers, which could further result in severe complications such as blindness, deafness, ptosis, and permanent facioplegia. Hence, how to ensure pain relief and reduce complications is the focus of clinical attention of temperature selection. Therefore, a lower temperature was recommended not only to ensure the therapeutic effects but also to reduce complications. In this study, we analyzed all studies on the selection of radiofrequency treatments at different temperatures and studied the patients' satisfaction degree of postoperative efficiency. We found that the temperature range of $68^{\circ} \mathrm{C}-70^{\circ} \mathrm{C}$ was better in patients' satisfaction 


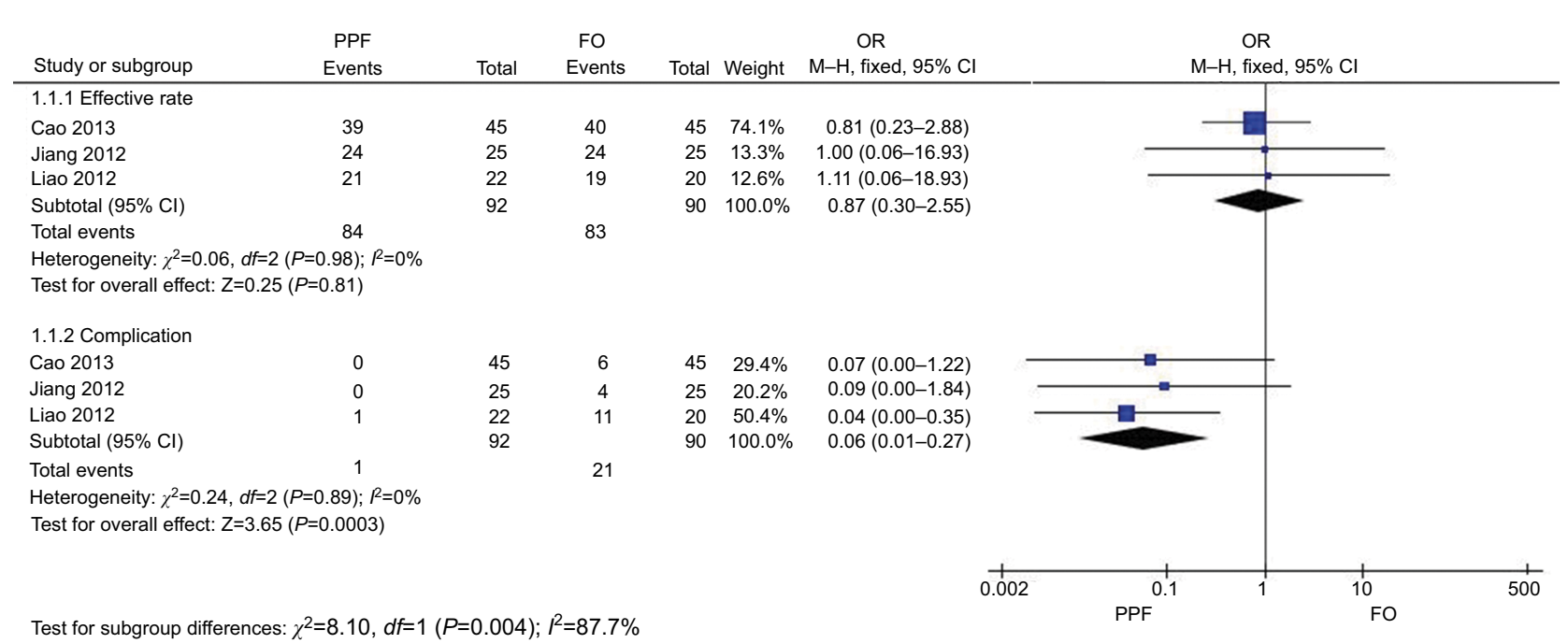

Figure 7 Comparison of the efficiency and safety between the PPF and FO routes in TN treatment via CRF.

Abbreviations: CRF, continuous radiofrequency; FO, foramen oval; PPF, pterygopalatine fossa; TN, trigeminal neuralgia.

\begin{tabular}{|c|c|c|c|c|c|c|c|c|c|}
\hline Study or subgroup & $\begin{array}{l}\text { FR route } \\
\text { Events }\end{array}$ & Total & $\begin{array}{l}\text { FO route } \\
\text { Events }\end{array}$ & Total & Weight & $\begin{array}{c}\text { OR } \\
M-H, \text { fixed, } 95 \% \mathrm{Cl}\end{array}$ & $\mathrm{M}-\mathrm{H}$, fixec & $\begin{array}{l}\mathrm{R} \\
\mathrm{d}, 95 \% \mathrm{Cl}\end{array}$ & \\
\hline \multicolumn{10}{|l|}{ 2.1.1 Effective rate } \\
\hline Chen 2017 & 28 & 30 & 29 & 30 & $69.6 \%$ & $0.48(0.04-5.63)$ & & & \\
\hline Huang 2015 & 20 & 20 & 17 & 20 & $15.0 \%$ & $8.20(0.40-169.90)$ & & & \\
\hline Zhao 2015 & 36 & 36 & 30 & 33 & $15.5 \%$ & $8.38(0.42-168.59)$ & & & \\
\hline Subtotal $(95 \% \mathrm{Cl})$ & & 86 & & 83 & $100.0 \%$ & $2.86(0.75-10.96)$ & & & \\
\hline Total events & 84 & & 76 & & & & & & \\
\hline \multicolumn{10}{|c|}{ Heterogeneity: $\chi^{2}=2.97, d f=2(P=0.23) ; l^{2}=33 \%$} \\
\hline \multicolumn{10}{|c|}{ Test for overall effect: $Z=1.53(P=0.13)$} \\
\hline \multicolumn{10}{|l|}{ 2.1.2 Complication } \\
\hline Chen 2017 & 0 & 30 & 5 & 30 & $11.1 \%$ & $0.08(0.00-1.44)$ & 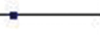 & - & \\
\hline Huang 2015 & 0 & 20 & 17 & 20 & $35.1 \%$ & $0.00(0.00-0.10) \longleftarrow$ & & & \\
\hline Zhao 2015 & 0 & 36 & 25 & 33 & $53.8 \%$ & $0.00(0.00-0.08) \leftarrow$ & & & \\
\hline Subtotal $(95 \% \mathrm{Cl})$ & & 86 & & 83 & $100.0 \%$ & $0.01(0.00-0.07)$ & & & \\
\hline Total events & 0 & & 47 & & & & & & \\
\hline \multicolumn{10}{|c|}{ Heterogeneity: $\chi^{2}=2.28, d f=2(P=0.32) ; l^{2}=12 \%$} \\
\hline & & & & & & 0.001 & $\begin{array}{l}0.1 \\
\text { FR route }\end{array}$ & ${ }^{1}$ FO route & 1000 \\
\hline
\end{tabular}

Figure 8 Comparison of the efficiency and safety between the FR route and FO route in TN treatment via CRF.

Abbreviations: CRF, continuous radiofrequency; FO, foramen oval; FR, foramen rotundum; $T N$, trigeminal neuralgia.

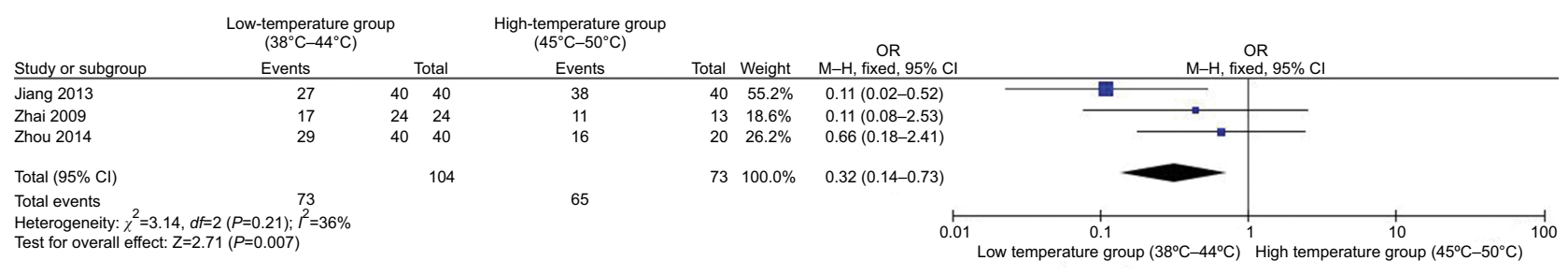

Figure 9 Comparison of the efficiency between different temperatures in TN treatment via PRF.

Abbreviations: PRF, pulsed radiofrequency; TN, trigeminal neuralgia.

degree, and the efficiency was better at $66^{\circ} \mathrm{C}-80^{\circ} \mathrm{C}$. When the temperatures were lower between two groups, the effect was better, which was associated with the previous reports. Yao et $\mathrm{al}^{30}$ pointed out that during the long-term follow-up, patients' satisfaction degree decreased with the occurrence of pain recurrence and complications. This finding suggests that postoperative complications and patient satisfaction should be taken into account during the pursuit of a greater 


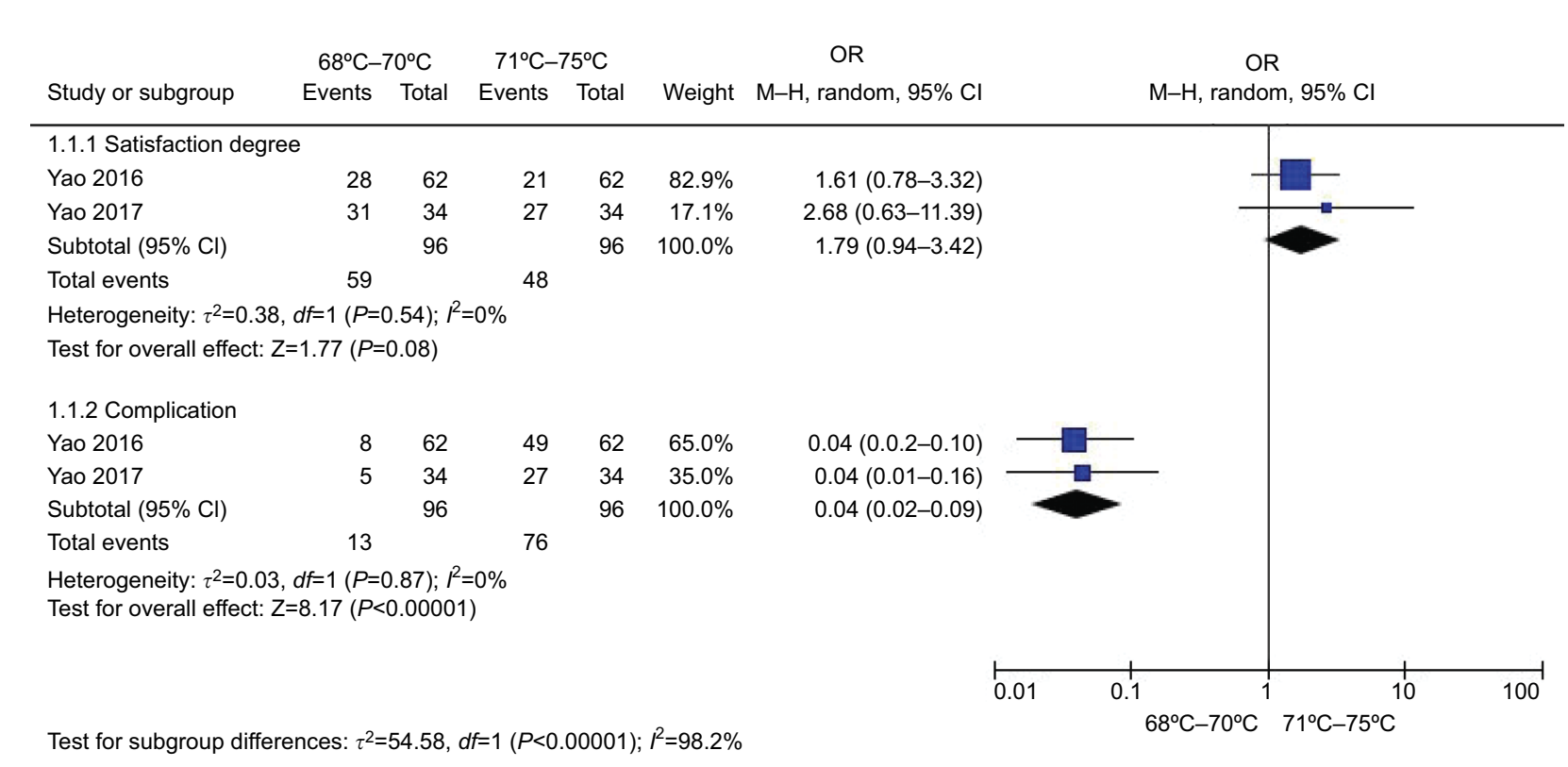

Figure 10 Comparison of the efficiency and safety between different temperatures $\left(68^{\circ} \mathrm{C}-70^{\circ} \mathrm{C}\right.$ vs $\left.71^{\circ} \mathrm{C}-75^{\circ} \mathrm{C}\right)$ in $\mathrm{TN}$ treatment via $\mathrm{CRF}$.

Abbreviations: CRF, continuous radiofrequency; TN, trigeminal neuralgia.

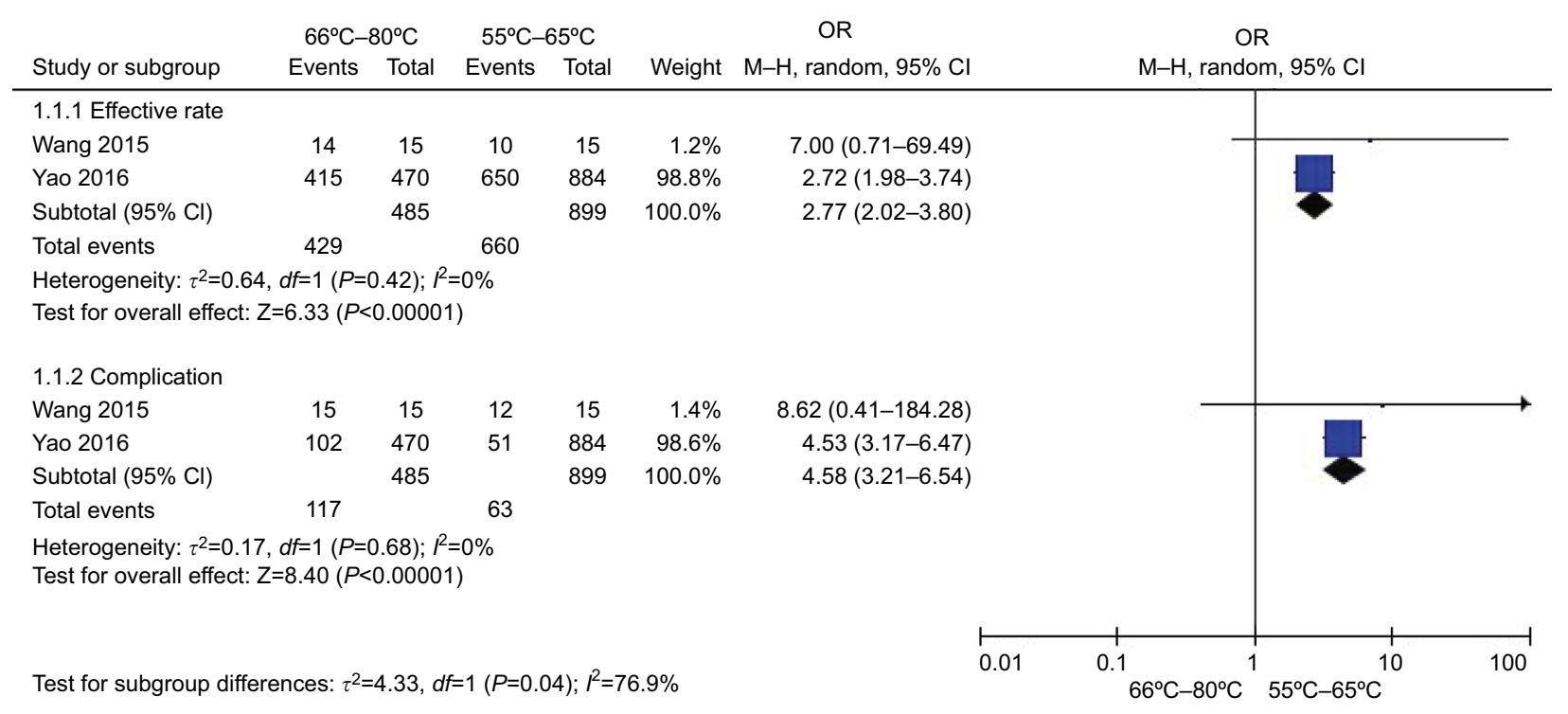

Figure I I Comparison of the efficiency and safety between different temperatures $\left(55^{\circ} \mathrm{C}-65^{\circ} \mathrm{C}\right.$ vs $\left.66^{\circ} \mathrm{C}-80^{\circ} \mathrm{C}\right)$ in TN treatment via $\mathrm{CRF}$.

Abbreviations: CRF, continuous radiofrequency; TN, trigeminal neuralgia.

pain relief. In terms of PRF treatment, there were few reports about the effect of different temperatures on the analgesic effect. In our study, we found that higher temperature group $\left(45^{\circ} \mathrm{C}-50^{\circ} \mathrm{C}\right)$ has a greater effect on treatment. While Zhou et $\mathrm{al}^{14}$ and Zhai et $\mathrm{al}^{15}$ showed that different temperatures $\left(38^{\circ} \mathrm{C}, 42^{\circ} \mathrm{C}, 45^{\circ} \mathrm{C}\right.$, and $\left.48^{\circ} \mathrm{C}\right)$ via $P R F$ were all effective in the patients, but increasing the temperature of PRF did not improve the analgesic effect and maintenance time. Moreover, Jiang et $\mathrm{al}^{50}$ showed that $45^{\circ} \mathrm{C}-50^{\circ} \mathrm{C}$ was a suggested temperature range for PRF, especially for the elders.
Different anatomic routes might have different impacts on RF efficiency. In this study, the RF efficiency between PPF and FO routes had no significant difference, and they both had the advantages of simple positioning, operation, and low recurrence rate in RF treatment. A number of clinical studies ${ }^{55,56}$ showed that RF thermocoagulation targeting on V2 branch could affect the ophthalmic and mandibular nerve function, while the PPF or FR route is safer with less postoperative complications and the possible reason is that the PPF or FR route is turning the target 


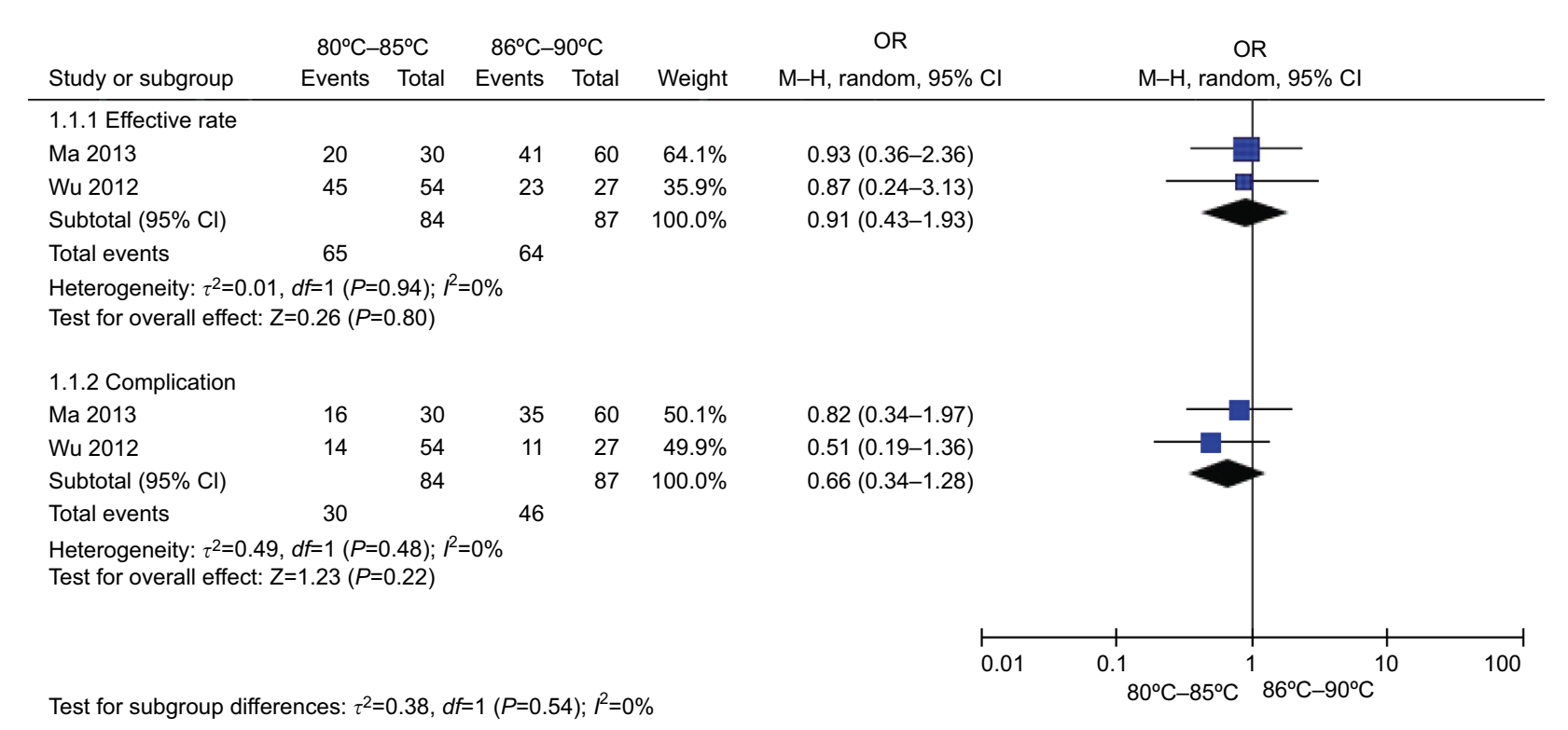

Figure 12 Comparison of the efficiency and safety between different temperatures $\left(80^{\circ} \mathrm{C}-85^{\circ} \mathrm{C}\right.$ vs $\left.86^{\circ} \mathrm{C}-90^{\circ} \mathrm{C}\right)$ in TN treatment via CRF. Abbreviations: CRF, continuous radiofrequency; TN, trigeminal neuralgia.

of RF thermocoagulation from the semilunar ganglion to the cranial branch of V2, from intracranial operation to extracranial operation, which obviously reduces the damage to the arteria meningea media, optic nerve, and other branches of the trigeminal nerve during the process of RF puncturing and thermocoagulation. ${ }^{43,49}$ Ding $^{57}$ showed that the FO route through the mandibular angle approach was reasonable too, with a higher target selectivity and a lower long-term pain recurrence rate. Furthermore, Chen et al ${ }^{58}$ indicated that the best approach of percutaneous puncturing of RF thermocoagulation for treating V2 in TN was the upper side against zygomatic and the inner side against the wall of maxillary sinus.

Despite of the main findings, there are some limitations in this study. First, the number of data samples was small, and some of the included articles were reported from the same institutes, which will inevitably result in a repetition and publication bias. Second, the long-term follow-up data of therapeutic effect in different studies were inconsistent. Therefore, we cannot get a uniform standard in the subgroup analysis. Third, most of the clinical trials included in this study were conducted in People's Republic of China, which may restrict the generalization of our conclusion. Fourth, the quality of the included studies was relatively low, which might limit the accountability of our results. Moreover, the evaluation criteria in response to the treatment effect are heterogeneous among different studies, some of them adopt patients' satisfaction degree, and others used an effective rate. In addition, some data are missing during the process of data extraction, limiting the robustness of this analysis.

\section{Conclusion}

CCPRF could achieve a greater efficacy and safety on TN treatment compared with PRF and CRF. Although there was no remarkable difference among PRF, FR, and FO routes in TN puncture treatment via CRF, the first two routes are safer. With regard to the guidance, 3D-printed template guide was more accurate for RF puncture than for imageonly guidance even with skin stimulation potential and semiconductor laser locator. Medium temperature range was better for CRF therapy, and higher temperature was recommended for PRF, especially for the elders. Further international multicenter RCTs comparing the effect and safety between PRF and CCPRF in terms of temperature, guidance, and routes are needed to confirm the evidence.

\section{Acknowledgments}

This work was supported by the grant from Six Talent Peaks Project in Jiangsu Province (2015-WSW-055), and Excellent Key Teachers in the "Qing Lan Project" of Jiangsu Colleges and Universities. We thank Shaswata Shoshi and Saharan Divya for proofreading the article.

\section{Disclosure}

The authors report no conflicts of interest in this work. 


\section{References}

1. van Hecke O, Austin SK, Khan RA, Smith BH, Torrance N. Neuropathic pain in the general population: a systematic review of epidemiological studies. Pain. 2014;155(4):654-662.

2. Ko AL, Ozpinar A, Lee A, Raslan AM, McCartney S, Burchiel KJ. Long-term efficacy and safety of internal neurolysis for trigeminal neuralgia without neurovascular compression. J Neurosurg. 2015;122(5):1048-1057.

3. Asplund P, Blomstedt P, Bergenheim AT. Percutaneous balloon compression vs percutaneous retrogasserian glycerol rhizotomy for the primary treatment of trigeminal neuralgia. J Neurosurg. 2016;78(3):421-428.

4. Régis J, Tuleasca C, Resseguier N, et al. Long-term safety and efficacy of Gamma Knife surgery in classical trigeminal neuralgia: a 497-patient historical cohort study. J Neurosurg. 2016;24(1):1079-1087.

5. Teixeira MJ, Siqueira SR, Almeida GM. Percutaneous radiofrequency rhizotomy and neurovascular decompression of the trigeminal nerve for the treatment of facial pain. Arq Neuropsiquiatr. 2006;64(4):983-989.

6. Yao P, Hong T, Wang ZB, et al. Treatment of bilateral idiopathic trigeminal neuralgia by radiofrequency thermocoagulation at different temperatures. Medicine (Baltimore). 2016;95(29):e4274.

7. Huang B, Yao M, Feng Z, et al. CT-guided percutaneous infrazygomatic radiofrequency neurolysis through foramen rotundum to treat V2 trigeminal neuralgia. Pain Med. 2014;15(8):1418-1428.

8. Bogduk N, Macintosh J, Marsland A. Technical limitations to the efficacy of radiofrequency neurotomy for spinal pain. Neurosurgery. 1987;20(4):529-535.

9. Zundert JV, Brabant S, de Kelft EV, Vercruyssen A, Buyten J-PV. Pulsed radiofrequency treatment of the Gasserian ganglion in patients with idiopathic trigeminal neuralgia. Pain. 2003;104(3):449-452.

10. Fang L, Tao W, Jingjing L, Nan J. Comparison of high-voltage- with standard-voltage pulsed radiofrequency of Gasserian ganglion in the treatment of idiopathic trigeminal neuralgia. Pain Pract. 2015;15(7):595-603.

11. Chua NH, Halim W, Beems T, Vissers KC. Pulsed radiofrequency treatment for trigeminal neuralgia. Anesth Pain Med. 2012;1(4):257-261.

12. Thapa D, Ahuja V, Dass C, Verma P. Management of refractory trigeminal neuralgia using extended duration pulsed radiofrequency application. Pain Physician. 2015;18(3):E433-435.

13. Jiang YH, Xia LJ, Wang J. Clinical effect of C arm guided pulsed radiofrequency in the treatment of senile trigeminal neuralgia. J Gerontol. 2013:1005-9202. Chinese.

14. Zhou C, Yang H, Huang XX. Effects of different temperatures on the efficacy of pulsed radiofrequency in the treatment of trigeminal neuralgia. J Youjiang Med Univ Nationalities. 2014;36(02):209-210. Chinese.

15. Zhai LP, Zh L, Chen JS. Influences of different temperature on clinical effect of percutaneous pulsed radiofrequency for the treatment of trigeminal neuralgia. Guangdong Medical J. 2009;30(2):206-208. Chinese.

16. Simopoulos TT, Kraemer J, Nagda JV, Aner M, Bajwa ZH. Response to pulsed and continuous radiofrequency lesioning of the dorsal root ganglion and segmental nerves in patients with chronic lumbar radicular pain. Pain Physician. 2008;11(2):137-144.

17. Headache Classification Subcommittee of the International Headache Society. The International classification of headache disorders. 2nd ed. Cephalalgia. 2004;24 (Suppl 1):9-160.

18. Headache Classification Committee of the International Headache Society (IHS). The International Classification of Headache Disorders, 3rd edition (beta version). Cephalalgia. 2013;33(9):629-808.

19. Jadad AR, Moore RA, Carroll D, et al. Assessing the quality of reports of randomized clinical trials: is blinding necessary? Control Clin Trials. 1996;17(1):1-12.

20. Li X, Ni J, Yang L, et al. A prospective study of Gasserian ganglion pulsed radiofrequency combined with continuous radiofrequency for the treatment of trigeminal neuralgia. J Clin Neurosci. 2012;19(6):824-828.
21. Ma ZW. Efficacy and side effects of different radiofrequency thermocoagulation on primary trigeminal neuralgia. Med and pharm. 2013;3(1):180-181. Chinese.

22. Wang ZK, Chen YM, Wang DQ. Therapeutic effect of different radiofrequency treatments on trigeminal neuralgia. Xinjiang Med. 2015;45(11):1645-1647.

23. Erdine S, Ozyalcin NS, Cimen A, Celik M, Talu GK, Disci R. Comparison of pulsed radiofrequency with conventional radiofrequency in the treatment of idiopathic trigeminal neuralgia. Eur J Pain. 2007;11(3):309-313.

24. Huibin Q, Jianxing L, Guangyu H, Dianen F. The treatment of first division idiopathic trigeminal neuralgia with radiofrequency thermocoagulation of the peripheral branches compared to conventional radiofrequency. J Clin Neurosci. 2009;16(11):1425-1429.

25. Yao P, Hong T, Zhu YQ, et al. Efficacy and safety of continuous radiofrequency thermocoagulation plus pulsed radiofrequency for treatment of V1 trigeminal neuralgia: A prospective cohort study. Medicine. 2016;95(44):e5247-5252.

26. Yao P, Deng Y, Hong T. Radiofrequency thermocoagulation for V2/V3 idiopathic trigeminal neuralgia: effect of treatment temperatures on long-term clinical outcomes: a cohort study. Medicine. 2016;95(26):4019-4028.

27. Elawamy A, Abdalla E, Shehata GA. Effects of pulsed versus conventional versus combined radiofrequency for the treatment of trigeminal neuralgia: a prospective study. Pain Physician. 2017;20(6): 873-881.

28. Kim JH, Yu HY, Park SY, Lee SC, Kim YC. Pulsed and conventional radiofrequency treatment: which is effective for dental procedure-related symptomatic trigeminal neuralgia? Pain Med. 2013;14(3):430-435.

29. Zhao WX, Wang Q, He MW, Yang LQ, Wu BS, Ni JX. Radiofrequency thermocoagulation combined with pulsed radiofrequency helps relieve postoperative complications of trigeminal neuralgia. Genet Mol Res. 2015;14(3):7616-7623.

30. Yao P, Hong T, Wang ZB, et al. Treatment of bilateral idiopathic trigeminal neuralgia by radiofrequency thermocoagulation at different temperatures. Medicine. 2016;95(29):e4274-4279.

31. Li XY, Ni JX, Yang LQ. The efficacy of gasserian ganglion combined radiofrequency treatment on trigeminal neuralgia. $J$ Rehabil Med. 2011;26(3):240-244. Chinese.

32. $\mathrm{Hu} \mathrm{B}$, Wang JG. Comparison of pulsed radiofrequency with radiofrequency thermocoagulation in the treatment of idiopathic trigeminal neuralgia. Int Med Health Guid News. 2008;14(15):11-13.

33. Meng L, Ma GF, Wang BG. Effect of pulsed radiofrequency on trigeminal neuralgia. Chin J Rehabil Theory Pract. 2009;15(7):611-612. Chinese.

34. Han Y, Wang R, Niu HX. Application of 3D printing navigational template in puncturing of foramen rotundum for radiofrequency thermocoagulation to treat primary trigeminal neuralgia(V2). Clin Anesthesiol. 2017;33(3):226-230. Chinese.

35. Lu LY, Han Y, Huai HB.3D printing puncture navigation moduleguided percutaneous radiofrequency thermocoagulation for treatment of trigeminal neuralgia. Chinese J Pain Med. 2015;21(12):914-918. Chinese.

36. Nie F, Su D, Shi Y, et al. A prospective study of X-ray imaging combined with skin stimulation potential-guided percutaneous radiofrequency thermocoagulation of the Gasserian ganglion for treatment of trigeminal neuralgia. Pain Med. 2014;15(9):1464-1469.

37. Nie FC, Su D, Chen JM. Trigeminal semilunar ganglion radiofrequency ablation guided by X-ray image combined with derma electrograph. Chinese J Pain Med. 2008;14(1):6-8. Chinese.

38. Qu PS, Tao F, Zheng HG.Effect of semiconductors lasers orientator on assistance of CT-guided puncture for the treatment of trigeminal neuralgia. Chin Gen Pract. 2009;12(12):1105-1106. Chinese.

39. Yang P, Li Y, Zhu J. Functional localization combined with CT location radiofrequency thermocoagulation for the treatment of primary trigeminal neuralgia. Pract Med J. 2011;27(24):4421-4423. Chinese. 
40. Chang HQ, Liao JX, Hou GY. Trigeminal peripheral branches radiofrequency thermocoagulation under the guide of $3 \mathrm{D}$ computerized tomography for the treatment of idiopathic trigeminal neuralgia. J Oral Maxillofac Surg. 2008;18(4):269-272. Chinese.

41. Wang DM, Lei JR, Zhu L. Percutaneous radiofrequency ablation of semilunar ganglion through foramen ovale under three-dimensional CT for treatment of trigeminal neuralgia. J Reg Anat Oper Surg. 2017;26(1): 58-61.

42. Fu S, Ps Q, Fang J. A new semiconductor laser locator assisted CT guided puncture effect. J Pract Med. 2011;27(1):56-58. Chinese.

43. Cao QW, Hq X, Cheng YG. Clinical comparison of radiofrequency thermocoagulation treatment of maxillary nerve in patients with idiopathic trigeminal neuralgia via pterygopalatine fossa and foramen ovale. Chinese J Pain Med. 2013;19(05):282-285. Chinese.

44. Huang BY, Wang S, Lin S. Clinical research of specific percutaneous radiofrequency thermocoagulation in the treatment of V2 trigeminal neuralgia. J Clin Stomatol. 2015;31(11):696-698.

45. Chen DL, Wang X, HeYQ, Li XL. Curative effect analysis of the radiofrequency thermocoagulation for treating maxillary nerve neuralgia by two percutaneous puncture methods under X-ray. Stomatology. 2017;37(10): 905-909. Chinese.

46. Jiang J, Liao X, Luo YH. Clinical efficacy of radiofrequency thermocoagulation treatment of maxillary nerve via sphenopalatine fossa or foramen ovale on primary trigeminal neuralgia. Chinese J Pain Med 2012;18(10):609-613. Chinese.

47. Liao MJ, Zou C, He YW. Comparison of radiofrequency thermocoagulation via two approaches guided by DSA in the treatment of primary trigeminal neuralgia branch II. Pain Clin J. 2012;8(3):171-176. Chinese.

48. Yao P, Wang ZB, Hong T. Comparative observation of bilateral trigeminal neuralgia after radiofrequency thermocoagulation at different temperatures. Chinese J Pain Med. 2017;23(1):33-38. Chinese.
49. Zhao L, Song T. Comparison of effect of CT guided percutaneous puncturing through foramen rotundum and oval foramen for radiofrequency thermocoagulation to treat primary trigeminal maxillary neuralgia. $J$ Chin Med Univ. 2015;44(04):311-314. Chinese.

50. Jiang YH, Xia LJ, Wang J. C type arm guided pulsed radiofrequency effect in the treatment of trigeminal neuralgia in elderly. Chin J Gerontol. 2013;33(14):3503-3504. Chinese.

51. Wu F, Chen SY, Zou YK. Clinical study of CT guided radiofrequency thermocoagulation for trigeminal neuralgia. Foreign Med Treat. 2012;31(30):166-167. Chinese.

52. Yadav YR, Nishtha Y, Sonjjay P, Vijay P, Shailendra R, Yatin K. Trigeminal Neuralgia. Asian J Neurosurg. 2017;12(4):585-597.

53. Sweet WH, Wepsic JG. Controlled thermocoagulation of trigeminal ganglion and rootlets for differential destruction of pain fibers. I Trigeminal neuralgia. J Neurosurg. 1974;40:143-156.

54. Sluijter ME, Cosman ER, Rittman WB III, Van Kleef M. The effects of pulsed radiofrequency fields applied to the dorsal root ganglion: A preliminary report. Pain Clin. 1998;11(2):109-117.

55. Qiao L, Zhu HW, Tao W. Neuronavigation guided radiofrequency thermocoagulation for trigeminal trigeminal ganglia oval puncture technique. Chinese J Pain Med. 2012;18(10):635-637. Chinese.

56. Zhang WC, Zhong WX, Li ST, Zheng XS, Yang M, Shi J. Neuronavigator-guided percutaneous radiofrequency thermocoagulation in the treatment of trigeminal neuralgia. Ir J Med Sci. 2012;181(1):7-13.

57. Ding WH. Application of the radiofrequency thermocoagulation of gasserian ganglion by percutaneous foramen ovale puncture from the gonial angle. J Zhejiang Univ. 2015. Chinese.

58. Chen ZW, Ma JB, Xie KY, et al. A study of the relations of foramen rotundum structure direction and the approach of percutaneous puncturing of radiofrequency thermocoagulation for treating V2 of primary trigeminal neuralgia. Zhonghua Yi Xue Za Zhi. 2018;98(6):436-443. Chinese.
Journal of Pain Research

\section{Publish your work in this journal}

The Journal of Pain Research is an international, peer reviewed, open access, online journal that welcomes laboratory and clinical findings in the fields of pain research and the prevention and management of pain. Original research, reviews, symposium reports, hypothesis formation and commentaries are all considered for publication

\section{Dovepress}

The manuscript management system is completely online and includes a very quick and fair peer-review system, which is all easy to use. Visit http://www.dovepress.com/testimonials.php to read real quotes from published authors. 